\title{
COMPLETE REDUCIBILITY OF THE PSEUDOVARIETY LSI
}

\author{
JOSÉ CARLOS COSTA \\ Centro de Matemática, Universidade do Minho, \\ Campus de Gualtar, 4700-320 Braga, Portugal. \\ jcosta@math. uminho.pt \\ CONCEIÇÃO NOGUEIRA \\ Escola Superior de Tecnologia e Gestão, Instituto Politécnico de Leiria, \\ Campus 2, Morro do Lena, Alto Vieiro, 2411-901 Leiria, Portugal. \\ conceicao.nogueira@estg.ipleiria.pt
}

February 9, 2009

\begin{abstract}
In this paper we prove that the pseudovariety $\mathbf{L S l}$ of local semilattices is completely $\kappa$-reducible, where $\kappa$ is the implicit signature consisting of the multiplication and the $\omega$-power. Informally speaking, given a finite equation system with rational constraints, the existence of a solution by pseudowords of the system over LSI implies the existence of a solution by $\kappa$-words of the system over LSl satisfying the same constraints.
\end{abstract}

2000 Mathematics Subject Classification: 20M05, 20M07, 20M35.

Keywords: Semigroup; pseudovariety; pseudoword; system of equations; implicit signature; complete tameness; complete reducibility; local semillatice; infinite word.

\section{Introduction}

A semigroup pseudovariety (a class of finite semigroups closed under taking homomorphic images, subsemigroups, and finite direct products) is said to be decidable if there is an algorithm to test membership of a given finite semigroup in that pseudovariety. One of the main motivations to study decidability comes from its applications in computer science, where many questions are related with this membership problem. It is known that decidability is not preserved by some of the most common pseudovariety operators, such as semidirect product, block product, Mal'cev product, join and power [1, 20, 13. Since pseudovarieties are often obtained from other ones by using these operators, several authors have been exploring the idea of establishing stronger conditions which are expected to be useful in proving decidability of pseudovarieties [5, 21]. In this context several concepts were introduced, such as the notion of tameness introduced by Almeida and Steinberg as a tool for proving decidability of the membership problem for semidirect products of pseudovarieties [10, 11].

The tameness property is parameterized by an implicit signature $\sigma$, and we speak of $\sigma$-tameness. Each finite semigroup can be naturally interpreted as an algebra for 
the signature $\sigma$, and such algebras are called $\sigma$-semigroups. Given an alphabet $A$ and a pseudovariety $\mathbf{V}$, the $\mathbf{V}$-free $\sigma$-semigroup over $A$ is denoted by $\Omega_{A}^{\sigma} \mathbf{V}$ and its elements are called $\sigma$-terms over $\mathbf{V}$. A pseudovariety $\mathbf{V}$ is said to be $\sigma$-tame if the word problem for $\sigma$-terms over $\mathbf{V}$ is decidable, and if $\mathbf{V}$ is $\sigma$-reducible, which means, informally speaking, the following: if a finite graph equation system with rational constraints has a solution in the free profinite semigroup "modulo" $\mathbf{V}$, then it has also a solution given by $\sigma$-terms and satisfying the same constraints. More generally, when not only graph equation systems but every finite systems of $\sigma$-term equations are considered, $\mathbf{V}$ is said to be completely $\sigma$-reducible (and completely $\sigma$-tame when, furthermore, the word problem for $\sigma$-terms over $\mathbf{V}$ is decidable). This extension of the notion of reducibility, was defined by Almeida [5] mainly because various kinds of such more general systems appear when different pseudovariety operators are considered. Moreover, unlike reducibility, the complete reducibility property is inherited by the dual pseudovariety. The notion of complete reducibility was introduced independently by Rhodes and Steinberg [21], using the different terminology of inevitable substitutions, in a more general setting, that of pseudovarieties of relational morphisms and not just of semigroups.

The implicit signature which is most commonly encountered in the literature is the canonical signature $\kappa=\left\{a b, a^{\omega-1}\right\}$ consisting of two pseudowords: $a b$, representing semigroup multiplication, and $a^{\omega-1}$, the unary pseudoinverse which, evaluated on an element $s$ of a profinite semigroup, takes the value of the unique inverse of $s e$ in the maximal subgroup of the closed subsemigroup generated by $s$, where $e$ denotes the idempotent of that subgroup.

There are several examples of reducibility results in the literature but relatively few results of complete reducibility. We recall some of these results. It is known, for instance, that the pseudovariety $\mathbf{G}$ of all finite groups is $\kappa$-tame [12, 3, 10] but it is not completely $\kappa$-tame [16]. Whether $\mathbf{G}$ is completely $\sigma$-tame for some other signature $\sigma$ is still an open question. The pseudovariety $\mathbf{G}_{p}$ of all finite $p$-groups, with $p$ prime, is not $\kappa$-tame [10] but Almeida has exhibited an infinite implicit signature with respect to which it is tame [4]. The pseudovariety $\mathbf{A b}$ of all finite abelian groups is completely $\kappa$-tame [9]. For aperiodic examples, we should mention the pseudovarieties $\mathbf{J}$ and $\mathbf{R}$ of all finite semigroups in which, respectively, the Green relations $\mathcal{J}$ and $\mathcal{R}$ are trivial. The complete $\kappa$-tameness of $\mathbf{J}$ is proved explicitly in [5], but it is also implicit in the proofs of [22] as is the $\kappa$-tameness of $\mathbf{J} \vee \mathbf{G}$. The proof of complete $\kappa$-tameness of $\mathbf{R}$ was obtained recently [7, 8], while its $\kappa$-tameness had already been established by the same authors in [6], where several joins involving $\mathbf{R}$, namely $\mathbf{R} \vee \mathbf{G}$, were also shown to be $\kappa$-tame.

Let LSI be the pseudovariety of all finite semigroups which are locally semilattices, that is, semigroups $S$ such that $e S e \in \mathbf{S l}$ for all idempotents $e \in S$. Notice that LSl is the pseudovariety associated, via Eilenberg's correspondence, to the variety of locally testable languages, which as one recalls is formed by the languages $L$ whose membership of a given word $u$ in $L$ can be decided by considering the factors of a fixed length $n$ of $u$ and its prefix and suffix of length $n-1$. This pseudovariety is already known to be $\kappa$-tame [15]. A weaker property, the pointlike subsets of a finite semigroup being decidable with respect to LSl, was first proved in [23. In this paper, we extend the above mentioned work of the first author and Teixeira [15] by proving the complete $\kappa$-tameness of LSl.

This work is organized as follows. After a section of preliminaries, where we introduce some notation and review some basic results on semigroups, pseudovarieties and words, 
we present the notions of complete $\kappa$-reducibility and complete $\kappa$-tameness in Section 3 . Section 4 is devoted to recall the basic facts about the pseudovariety LSI, namely the solution of the $\kappa$-word problem for LSl, which are needed to prove the main result of the paper. We next present some technical results on combinatoric of words which are essential for our purposes. Finally, Section 6 gives the proof of the complete $\kappa$ reducibility of $\mathbf{L S l}$.

\section{Preliminaries}

In this section, we briefly recall notation, basic definitions and results that will be needed in the following sections. The reader is referred to [2, 5] for general background about the classical theories of semigroups, pseudovarieties and profinite semigroups and to [17. for further details about combinatorics on words.

\subsection{Words}

Throughout this paper, $A$ denotes a finite non-empty set called alphabet. The free semigroup (resp. the free monoid) generated by $A$ is denoted by $A^{+}$(resp. $A^{*}$ ). The length of a word $w \in A^{*}$ is denoted by $|w|$. The empty word is denoted by 1 and its length is 0 . The following result is known as Fine and Wilf's Theorem (see [17]).

Proposition 2.1 Let $u, v \in A^{+}$. If two powers $u^{k}$ and $v^{n}$ of $u$ and $v$ have a common prefix (resp. suffix) of length at least $|u|+|v|-\operatorname{gcd}(|u|,|v|)$, then $u$ and $v$ are powers of the same word.

A word $w \in A^{+}$is said to be primitive if it is not a power of another word; that is, if $w=u^{n}$ for some $u \in A^{*}$ and $n \geq 1$ implies $w=u$ (and $n=1$ ). Two words $w$ and $z$ are said to be conjugate if there exist words $u, v \in A^{*}$ such that $w=u v$ and $z=v u$. We notice that, if $w$ is a primitive word and $z$ is a conjugate of $w$, then $z$ is also primitive. Let an order be fixed for the letters of the alphabet A. A Lyndon word is a primitive word which is minimal, with respect to the lexicographic ordering, in its conjugation class.

A bi-infinite (resp. right-infinite, left-infinite) word on $A$ is a sequence $w=\left(a_{n}\right)_{n}$ of letters of $A$ indexed by $\mathbb{Z}$ (resp. $\mathbb{N},-\mathbb{N}$ ). We denote $w(n)=a_{n}$ and say that $a_{n}$ is the letter of $w$ at position $n$. The sets of bi-infinite, right-infinite and left-infinite words on $A$ will be denoted, respectively, by $A^{\mathbb{Z}}, A^{\mathbb{N}}$ and $A^{-\mathbb{N}}$.

Let $w$ be a word. For integers $i$ and $j$ such that $i \leq j$, we denote by $w[i, j]$ the word $a_{i} \cdots a_{j}$. In case $w \in A^{\mathbb{Z}}$ (resp. $w \in A^{\mathbb{N}}$ ) and $i \in \mathbb{Z}$ (resp. $i \in \mathbb{N}$ ), we denote by $w\left[i,+\infty\left[\right.\right.$ the right-infinite word $a_{i} a_{i+1} \cdots$ and say that this word is a suffix of $w$. Dually, if $w \in A^{\mathbb{Z}}$ (resp. $w \in A^{-\mathbb{N}}$ ) and $i \in \mathbb{Z}$ (resp. $i \in-\mathbb{N}$ ), we denote by $\left.\left.w\right]-\infty, i\right]$ the left-infinite word $\cdots a_{i-1} a_{i}$ and say that this word is a prefix of $w$.

A word $x \in A^{*}$ is a factor of a word $w$, and $w$ is an extension of $x$, if $x=1$ or $x=w[i, j]$, for some integers $i$ and $j$ with $i \leq j$. In such case, $w[i, j]$ is said to be an occurrence of the factor $x$ in $w$. We say that two occurrences $w[i, j]$ and $w[k, l]$ of factors in a word $w$ are disjoint (or that they do not overlap) if the integer intervals $[i, j]$ and $[k, l]$ are disjoint sets. For each pair of words $w, x \in A^{*}$, we denote by $\operatorname{occ}(x, w)$ the number of occurrences of $x$ in $w$, and by $\operatorname{docc}(x, w)$ the maximal number of disjoint occurrences of $x$ in $w$. 
We let

$$
A^{\infty}=A^{+} \cup A^{\mathbb{N}} \text { and } A^{-\infty}=A^{+} \cup A^{-\mathbb{N}} .
$$

The product of two elements $w, z$ of $A^{\infty}$ is defined as follows: if $w, z \in A^{+}$, then $w z$ is defined in the usual way; right-infinite words are left zeros; finally, if $w$ is finite and $z$ is a right-infinite word, then $w z$ is a right-infinite word defined by

$$
(w z)_{n}=\left\{\begin{array}{cc}
w_{n} & \text { if } n \leq|w| \\
z_{n-|w|} & \text { otherwise. }
\end{array}\right.
$$

The product of elements of $A^{-\infty}$ is defined symmetrically. It is a straightforward observation that $A^{\mathbb{N}}, A^{-\mathbb{N}}, A^{\infty}$ and $A^{-\infty}$ are semigroups.

A word $x \in A^{*}$ is a prefix of a word $w \in A^{\infty}$, and $w$ is a right-extension of $x$, if there exists $z \in A^{\infty}$ such that $w=x z$. Dually, $x \in A^{*}$ is a suffix of $w \in A^{-\infty}$, and $w$ is a left-extension of $x$, if there exists $z \in A^{-\infty}$ such that $w=z x$.

A right-infinite word of the form $v u^{+\infty}=v u u u \cdots$, with $u \in A^{+}$and $v \in A^{*}$, is said to be ultimately periodic and $u$ is said to be a period of $v u^{+\infty}$. Each ultimately periodic word $w \in A^{\mathbb{N}}$ admits a unique representation $w=v u^{+\infty}$, called the normal form of $w$, such that $u$ is a Lyndon word and $u$ is not a suffix of $v$. Ultimately periodic left-infinite words are defined symmetrically as being words of the form $u^{-\infty} v=\cdots u u u v$. Each ultimately periodic word $w \in A^{-\mathbb{N}}$ admits a unique representation $w=u^{-\infty} v$, called the normal form of $w$, such that $u$ is a Lyndon word and $u$ is not a prefix of $v$. An ultimately periodic word $w \in A^{\mathbb{N}}$ (resp. $w \in A^{-\mathbb{N}}$ ) which can be written in the form $w=u^{+\infty}$ (resp. $w=u^{-\infty}$ ) for some $u \in A^{+}$, is said to be periodic.

When writing a specific bi-infinite word, we need to specify which is the letter at position 0. We do this by putting a "." on the left of the letter. For instance, given words $x=\left(x_{i}\right)_{i \in-\mathbb{N}} \in A^{-\mathbb{N}}$ and $y=\left(y_{i}\right)_{i \in \mathbb{N}} \in A^{\mathbb{N}}$, we denote by $x \cdot y$ the bi-infinite word $w=\cdots x_{-2} x_{-1} \cdot y_{1} y_{2} \cdots$. A bi-infinite word $w=x \cdot y$ is said to be left-ultimately periodic (resp. right-ultimately periodic) if the left-infinite word $x \in A^{-\mathbb{N}}$ (resp. the right-infinite word $y \in A^{\mathbb{N}}$ ) is ultimately periodic. The word $w$ is said to be ultimately periodic if it is both left-ultimately and right-ultimately periodic, and it is said to be periodic if one can choose $x=u^{-\infty}$ and $y=u^{+\infty}$ for some $u \in A^{+}$.

The shift operator on $A^{\mathbb{Z}}$ is the function $\sigma: A^{\mathbb{Z}} \rightarrow A^{\mathbb{Z}}$ which sends each $\left(a_{i}\right)_{i \in \mathbb{Z}}$ to $\left(a_{i+1}\right)_{i \in \mathbb{Z}}$. The relation $\sim$ on $A^{\mathbb{Z}}$ given by

$$
w \sim z \quad \text { if and only if } \exists n \in \mathbb{Z}, w=\sigma^{n}(z)
$$

is an equivalence relation. The equivalence class of an element $w \in A^{\mathbb{Z}}$ relative to $\sim$ is called the orbit of $w$ and is denoted by $\mathcal{O}(w)$. Most of the times in this paper, we will be interested in a bi-infinite word only because of its factors. Since any two -equivalent bi-infinite words have the same factors, we will usually not distinguish a bi-infinite word from its orbit.

It is well known that a bi-infinite word $w$ is periodic if and only if $w=\sigma^{n}(w)$ for some $n>0$ if and only if $\mathcal{O}(w)$ is a finite set. For instance, the orbit of the periodic word $w=(a b a)^{-\infty} \cdot(a b a)^{+\infty}$ is

$$
\mathcal{O}(w)=\left\{w,(b a a)^{-\infty} \cdot(b a a)^{+\infty},(a a b)^{-\infty} \cdot(a a b)^{+\infty}\right\} .
$$

Given a left-infinite word $x \in A^{-\mathbb{N}}$ and a right-infinite word $y \in A^{\mathbb{N}}$, we denote by $x y$ the orbit of the bi-infinite word $x \cdot y$. For $u \in A^{+}$, we denote $u^{\infty}=u^{-\infty} u^{+\infty}$. For instance, $(a b a)^{\infty}$ represents the orbit $\mathcal{O}(w)$ in $(2.1)$. 


\subsection{Implicit operations and implicit signatures}

For a semigroup $S$, we denote by $S^{1}$ the smallest monoid containing $S$. Given an element $s$ of a finite semigroup (resp. compact topological semigroup), the subsemigroup (resp. the closed subsemigroup) generated by $s$ contains a unique idempotent, denoted by $s^{\omega}$. Moreover, $s^{\omega-1}$ denotes the inverse of $s^{\omega+1}\left(=s^{\omega} s\right)$ in the maximal subgroup (resp. closed subgroup) containing $s^{\omega}$. As usual, we denote by $E(S)$ the set of idempotents of a semigroup $S$.

A proof of the following classical result can be found in [2, Proposition 3.7.1].

Lemma 2.2 Let $S$ be a finite semigroup and $k=|S|$. For any $s_{1}, \ldots, s_{k} \in S$, there exist integers $1 \leq i \leq j \leq k$, such that, $s_{1} \cdots s_{k}=s_{1} \cdots s_{i-1}\left(s_{i} \cdots s_{j}\right)^{\omega} s_{j+1} \cdots s_{k}$.

A morphism of semigroups is an application $\varphi: S \rightarrow T$ between semigroups $S$ and $T$ that respects the multiplication operation of the semigroups. A relational morphism of semigroups is a binary relation $\theta: S \rightarrow T$ between semigroups $S$ and $T$ with domain $\mathrm{S}$ which is a subsemigroup of $S \times T$.

By a profinite semigroup, we mean a compact and totally disconnected semigroup. Equivalently, profinite semigroups are projective limits of finite semigroups. For a pseudovariety $\mathbf{V}$, a pro- $\mathbf{V}$ semigroup is a projective limit of semigroups of $\mathbf{V}$. We consider finite sets endowed with discrete topology. For an alphabet $A$, the $A$-generated elements of $\mathbf{V}$ form a directed system and the respective projective limit will be denoted by $\bar{\Omega}_{A} \mathbf{V}$. Elements of $\bar{\Omega}_{A} \mathbf{V}$ are called pseudowords (over $\mathbf{V}$ ). The semigroup $\bar{\Omega}_{A} \mathbf{V}$ is the free semigroup over $A$ for the class of all pro- $\mathbf{V}$ semigroups, which means that, for each pro- $\mathbf{V}$ semigroup $S$ and each function $\varphi: A \longrightarrow S$, there is a unique continuous morphism $\bar{\varphi}: \bar{\Omega}_{A} \mathbf{V} \longrightarrow S$ extending $\varphi$. The mapping $\bar{\varphi}$ is also said to be induced by $\varphi$. In particular, if $A$ is a subset of a finite set $B$, then the unique continuous morphism $\bar{\Omega}_{A} \mathbf{V} \rightarrow \bar{\Omega}_{B} \mathbf{V}$ induced by the inclusion mapping $A \hookrightarrow B$ is injective. We will identify $\bar{\Omega}_{A} \mathbf{V}$ with its image and therefore view $\bar{\Omega}_{A} \mathbf{V}$ as the closed subsemigroup of $\bar{\Omega}_{B} \mathbf{V}$ generated by $A$. Moreover, this leads to a natural interpretation of the pseudowords as (A-ary) implicit operations: to each $\pi \in \bar{\Omega}_{A} \mathbf{V}$ is associated an operation $\pi_{S}: S^{A} \longrightarrow S$ which maps $\varphi \in S^{A}$ to $\bar{\varphi}(\pi)$. The subsemigroup of $\bar{\Omega}_{A} \mathbf{V}$ generated by $A$ is denoted by $\Omega_{A} \mathbf{V}$ and is a dense subsemigroup of $\bar{\Omega}_{A} \mathbf{V}$ whose elements are said to be finite pseudowords (or explicit operations) over $\mathbf{V}$. As important examples of implicit operations, we mention the binary implicit operation ab of multiplication, and the two unary implicit operations $a^{\omega}, \omega$-power, and $a^{\omega-1},(\omega-1)$-power, which associate to each element $s$, of a profinite semigroup $S$, the elements $s^{\omega}$ and $s^{\omega-1}$ of $S$, respectively.

The pseudovariety of all finite semigroups is denoted by $\mathbf{S}$ and, for each pseudovariety $\mathbf{V}, p_{\mathbf{V}}$ denotes the canonical projection from $\bar{\Omega}_{A} \mathbf{S}$ into $\bar{\Omega}_{A} \mathbf{V}$. In particular, we write $c$ instead of $p_{\mathbf{S l}}$, where $\mathbf{S l}$ is the pseudovariety of semilattices, and we call $c(\pi)$ the content of $\pi$. Recall that the semigroup $\bar{\Omega}_{A} \mathbf{S l}$ is isomorphic to $(\mathcal{P}(A), \cup)$ and $c(a)=\{a\}$ for all $a \in A$.

A pseudoidentity is a formal equality $\pi=\rho$ where $\pi, \rho \in \bar{\Omega}_{A} \mathbf{S}$ for some alphabet A. When $\pi, \rho \in \Omega_{A} \mathbf{S}, \pi=\rho$ is also called an identity. A finite semigroup $S$ satisfies a pseudoidentity $\pi=\rho$ if $\pi_{S}=\rho_{S}$. A pseudovariety $\mathbf{V}$ satisfies a pseudoidentity $\pi=$ $\rho$, written $\mathbf{V} \models \pi=\rho$, if every semigroup in $\mathbf{V}$ satisfies $\pi=\rho$, which means that $p_{\mathbf{V}}(\pi)=p_{\mathbf{V}}(\rho)$. By Reiterman's theorem [19], each pseudovariety is defined by a set $\Sigma$ of pseudoidentities. The pseudovariety defined by $\Sigma$ is denoted by $\llbracket \Sigma \rrbracket$. 
Given $\pi \in \bar{\Omega}_{A} \mathbf{S}$, we say that $\rho \in \bar{\Omega}_{A} \mathbf{S}$ is a factor (resp. a prefix, a suffix) of $\pi$ if there are $\pi_{1}, \pi_{2} \in\left(\bar{\Omega}_{A} \mathbf{S}\right)^{1}$ such that $\pi=\pi_{1} \rho \pi_{2}$ (resp. $\pi=\rho \pi_{2}, \pi=\pi_{1} \rho$ ). A bi-infinite word $w$ is a (bi-infinite) factor of $\pi$ if every finite word which is a factor of $w$ is also a factor of $\pi$. Notice that a bi-infinite word $w$ is a factor of $\pi$ if and only if every element of $\mathcal{O}(w)$ is a factor of $\pi$.

An implicit signature $\sigma$ is a set of implicit operations containing the multiplication. Every profinite semigroup has a natural structure of a $\sigma$-semigroup, via the interpretation of implicit operations as operations on profinite semigroups. For a pseudovariety $\mathbf{V}$, we denote by $\Omega_{A}^{\sigma} \mathbf{V}$ the free $\sigma$-semigroup generated by $A$ in the variety of $\sigma$-semigroups generated by $\mathbf{V}$. The elements of $\Omega_{A}^{\sigma} \mathbf{V}$ are called $\sigma$-terms over $\mathbf{V}$, and elements of $\Omega_{A}^{\sigma} \mathbf{S}$ are called $\sigma$-terms. We denote by $p_{\mathbf{V}}: \Omega_{A}^{\sigma} \mathbf{S} \longrightarrow \Omega_{A}^{\sigma} \mathbf{V}$ the morphism of $\sigma$-semigroups determined by the choice of generators. Notice that this notation is consistent since $\Omega_{A}^{\sigma} \mathbf{S}$ may be seen as a subsemigroup of $\bar{\Omega}_{A} \mathbf{S}$, whence this morphism is the restriction of $p_{\mathbf{V}}: \bar{\Omega}_{A} \mathbf{S} \rightarrow \bar{\Omega}_{A} \mathbf{V}$ to $\Omega_{A}^{\sigma} \mathbf{S}$. The $\sigma$-word problem for $\mathbf{V}$ consists in determining whether two $\sigma$-terms represent the same element of $\bar{\Omega}_{A} \mathbf{V}$.

The most commonly used signature is $\kappa=\left\{a b, a^{\omega-1}\right\}$. However, since we are going to work with the aperiodic pseudovariety $\mathbf{L S 1}$, which therefore satisfies $a^{\omega}=a^{\omega-1}$, in this paper we are going to work instead with the implicit signature $\left\{a b, a^{\omega}\right\}$, still denoted by $\kappa$.

\section{Complete reducibility}

We assume the reader is acquainted with the notion of reducibility. For an introduction to this notion and for more details, the reader is referred to [5, 8]. We start with the formal setup of the problem of complete reducibility of a system of equations for a generic pseudovariety $\mathbf{V}$ of semigroups.

Let $\sigma$ be an implicit signature and let $X$ and $P$ be finite disjoint sets. Elements of $X$ are called variables and elements of $P$ are called parameters. Let $S$ be a finite $A$-generated semigroup.

In general, we are given a finite system $\mathcal{S}$ of $\sigma$-equations

$$
u_{i}=v_{i} \quad(i=1, \ldots, h),
$$

where each of the pseudowords $u_{i}$ and $v_{i}$ belong to the semigroup $\Omega_{X \cup P}^{\sigma} \mathbf{S}$.

Assume that we are given the following mappings, pictured in the diagram

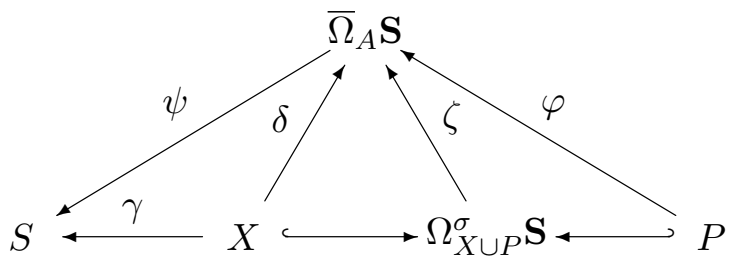

Solution $\delta$ and involved mappings

where:

- $\psi: \bar{\Omega}_{A} \mathbf{S} \rightarrow S$ is a continuous morphism that respects the choice of generators;

- $\gamma: X \rightarrow S$ gives a constraint in $S$ for each variable; 
- $\varphi: P \rightarrow \bar{\Omega}_{A} \mathbf{S}$ is an evaluation of the parameters such that $\varphi(P) \subseteq \Omega_{A}^{\sigma} \mathbf{S}$;

- $\delta: X \rightarrow \bar{\Omega}_{A} \mathbf{S}$ is an evaluation of the variables by pseudowords;

- $\zeta: \Omega_{X \cup P}^{\sigma} \mathbf{S} \rightarrow \bar{\Omega}_{A} \mathbf{S}$ is the $\sigma$-morphism defined by $\zeta_{\mid X}=\delta$ and $\zeta_{\mid P}=\varphi$.

We say that $\delta$ is a solution of the system $\mathcal{S}$ over $\mathbf{V}$ with respect to $(\varphi, \gamma, \psi)$ if

$$
\left\{\begin{array}{l}
\forall i \in\{1, \ldots, h\} \quad \mathbf{V} \models \zeta\left(u_{i}\right)=\zeta\left(v_{i}\right) \\
\psi \circ \delta=\gamma .
\end{array}\right.
$$

Furthermore, if $\delta(X) \subseteq \Omega_{A}^{\sigma} \mathbf{S}$, then $\delta$ is called a $\sigma$-solution of $\mathcal{S}$ over $\mathbf{V}$ with respect to $(\varphi, \gamma, \psi)$. The triple $(\varphi, \gamma, \psi)$ will be sometimes understood. If $P=\emptyset$, then we just speak about solutions with respect to $(\gamma, \psi)$. In this case, we will use the notation $\delta$ to represent both the evaluation of the variables and its extension to a $\sigma$-morphism $\Omega_{X}^{\sigma} \mathbf{S} \rightarrow \bar{\Omega}_{A} \mathbf{S}$.

A pseudovariety $\mathbf{V}$ is said to be $\sigma$-reducible for a system $\mathcal{S}$ if the existence of a solution $\delta$ of $\mathcal{S}$ over $\mathbf{V}$ with respect to a triple $(\varphi, \gamma, \psi)$ entails the existence of a $\sigma$-solution $\delta^{\prime}$ of $\mathcal{S}$ over $\mathbf{V}$ with respect to $(\varphi, \gamma, \psi)$. We say that $\mathbf{V}$ is completely $\sigma$-reducible if it is $\sigma$-reducible for every such system. If $\mathbf{V}$ is $\sigma$-reducible for every system of equations associated with finite graphs, then we say that $\mathbf{V}$ is $\sigma$-reducible.

In the language of [21, Chapter 3], where the same concept is developed, $\gamma$ is said to be a $\mathbf{V}$-inevitable substitution with respect to the system $\mathcal{S}$ if a solution $\delta$ exists.

We say that a recursively enumerable pseudovariety $\mathbf{V}$ is (completely) $\sigma$-tame if it is (completely) $\sigma$-reducible and the $\sigma$-word problem for $\mathbf{V}$ is decidable. Finally, we say that a pseudovariety is (completely) tame if it is (completely) $\sigma$-tame with respect to a recursively enumerable implicit signature $\sigma$ consisting of computable implicit operations.

\section{Implicit operations on LSl}

This section gathers some basic statements about the free pro-LSl semigroup.

\subsection{Basic properties of LSl}

Recall that $\mathbf{L S l}$ is the pseudovariety defined by the pseudoidentities

$$
x^{\omega} y x^{\omega} y x^{\omega}=x^{\omega} y x^{\omega}, \quad x^{\omega} y x^{\omega} z x^{\omega}=x^{\omega} z x^{\omega} y x^{\omega} .
$$

The pseudovarieties $\mathbf{K}=\llbracket x^{\omega} y=x^{\omega} \rrbracket$ and $\mathbf{D}=\llbracket y x^{\omega}=x^{\omega} \rrbracket$, of all finite semigroups whose idempotents are respectively left zeros and right zeros, are two important subpseudovarieties of LSl. Notice also that $\mathbf{L I}=\llbracket x^{\omega} y z^{\omega}=x^{\omega} z^{\omega} \rrbracket$, the pseudovariety of locally trivial semigroups, is the join of $\mathbf{K}$ and $\mathbf{D}$. This means that a pseudoidentity $\pi=\rho$ is satisfied by $\mathbf{L I}$ if and only if it is satisfied by both $\mathbf{K}$ and $\mathbf{D}$. Now we recall that, each $\mathbf{V} \in\{\mathbf{K}, \mathbf{D}, \mathbf{L I}, \mathbf{L S} \mathbf{S}\}$ does not satisfy any non-trivial identity, whence we may identify the free semigroup $A^{+}$with the subsemigroup $\Omega_{A} \mathbf{V}$ of $\bar{\Omega}_{A} \mathbf{V}$. Moreover $\bar{\Omega}_{A} \mathbf{K}$ and $\bar{\Omega}_{A} \mathbf{D}$ are isomorphic to $A^{\infty}$ and $A^{-\infty}$, respectively. In $\bar{\Omega}_{A} \mathbf{K}$, the right-infinite word $v u^{+\infty}$, where $v \in A^{*}$ and $u \in A^{+}$, corresponds to the pseudoword $v u^{\omega}$. A dual remark holds for $\mathbf{D}$. 
Let $n$ be a positive integer. We denote by $\equiv_{n}$ the congruence on $\bar{\Omega}_{A} \mathbf{S}$ given, for every $\pi, \rho \in \bar{\Omega}_{A} \mathbf{S}$, by

$$
\pi \equiv_{n} \rho \quad \text { if } \quad \pi \text { and } \rho \text { have the same prefix, suffix and factors of length } n .
$$

The following proposition summarizes some properties of the pseudoidentities satisfied by LSl. This result is an immediate consequence of [14, Theorem 3.3].

Proposition 4.1 Let $\pi, \rho \in \bar{\Omega}_{A} \mathbf{S}$. The following conditions are equivalent:

(i) $p_{\mathbf{L S I}}(\pi)=p_{\mathbf{L S I}}(\rho)$;

(ii) $\pi \equiv_{n} \rho$ for every $n \in \mathbb{N}$;

(iii) $p_{\mathbf{L I}}(\pi)=p_{\mathbf{L I}}(\rho)$ and $\pi$ and $\rho$ have the same finite factors;

(iv) $p_{\mathbf{L I}}(\pi)=p_{\mathbf{L I}}(\rho)$ and $\pi$ and $\rho$ have the same bi-infinite factors.

Moreover, if $\pi$ and $\rho$ are infinite pseudowords, then a bi-infinite word $\mathbf{w} \in A^{\mathbb{Z}}$ is a factor of $\pi \rho$ if and only if $\mathbf{w}$ is a factor of $\pi$ or a factor of $\rho$, or $\mathbf{w} \in \mathcal{O}(\overleftrightarrow{\pi \rho})$ where $\overleftrightarrow{\pi \rho}$ is the bi-infinite word $p_{\mathbf{D}}(\pi) \cdot p_{\mathbf{K}}(\rho)$.

As an immediate consequence, we have the following cancelation properties.

Corollary 4.2 Let $\pi_{1}, \pi_{2}, \rho \in \bar{\Omega}_{A} \mathbf{S}$ be such that $\mathbf{L S l}$ verifies $\rho \pi_{1}=\rho \pi_{2}$ or $\pi_{1} \rho=\pi_{2} \rho$. If $\rho$ is a finite word or $c(\rho)$ is disjoint with both $c\left(\pi_{1}\right)$ and $c\left(\pi_{2}\right)$, then $\mathbf{L S} 1=\pi_{1}=\pi_{2}$.

Another consequence of Proposition 4.1 is the following factorization property.

Lemma 4.3 Let $\pi_{1}, \ldots, \pi_{r}, \rho_{1}, \rho_{2} \in \bar{\Omega}_{A} \mathbf{S}$ be infinite pseudowords and suppose that LSl $\models \pi_{1} \cdots \pi_{r}=\rho_{1} \rho_{2}$. Then, either $\overleftrightarrow{\rho_{1} \rho_{2}} \sim \overleftrightarrow{\pi_{i} \pi_{i+1}}$ for some $i \in\{1, \ldots, r-1\}$, or there exists a $j \in\{1, \ldots, r\}$ and infinite pseudowords $\pi_{j}^{\prime}, \pi_{j}^{\prime \prime} \in \bar{\Omega}_{A} \mathbf{S}$ such that $\pi_{j}=\pi_{j}^{\prime} \pi_{j}^{\prime \prime}$ and $\overleftrightarrow{\rho_{1} \rho_{2}}=\overleftrightarrow{\pi_{j}^{\prime} \pi_{j}^{\prime \prime}}$

Proof. For each positive integer $k$ let $u_{k}$ and $v_{k}$ be, respectively, the suffix and the prefix of $p_{\mathbf{D}}\left(\rho_{1}\right)$ and $p_{\mathbf{K}}\left(\rho_{2}\right)$ of length $k$. Since $u_{k} v_{k}$ is a factor of $\rho_{1} \rho_{2}$ and $\mathbf{L S l}$ verifies

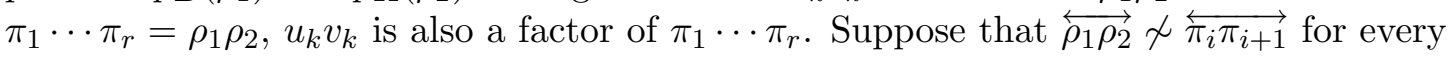
$i \in\{1, \ldots, r-1\}$. Then, there is a $j \in\{1, \ldots, r\}$ such that $u_{k} v_{k}$ is a factor of $\pi_{j}$ for every $k$. Hence, there is a factorization $\pi_{j}=x_{k} u_{k} v_{k} y_{k}$ for every $k$ and, since $\bar{\Omega}_{A} \mathbf{S}$ is compact, we may assume that both sequences $\left(x_{k} u_{k}\right)_{k}$ and $\left(v_{k} y_{k}\right)_{k}$ converge, say to $\pi_{j}^{\prime}$ and $\pi_{j}^{\prime \prime}$ respectively. Therefore $\pi_{j}=\pi_{j}^{\prime} \pi_{j}^{\prime \prime}$ and $\overleftrightarrow{\rho_{1} \rho_{2}}=p_{\mathbf{D}}\left(\rho_{1}\right) \cdot p_{\mathbf{K}}\left(\rho_{2}\right)=\overleftrightarrow{\pi_{j}^{\prime} \pi_{j}^{\prime \prime}}$.

A proof of the following simple, but fundamental, result can be found in [15].

Lemma 4.4 Let $S$ be a finite semigroup and let $n$ be a positive integer. If $\pi \in \bar{\Omega}_{A} \mathbf{S}$ is an infinite pseudoword, then there exists a word $w \in A^{+}$such that $w \equiv_{n} \pi$ and $S$ satisfies $w=\pi$.

The next result ([2, Corollary 5.6.2]) presents an useful decomposition of the infinite elements of the semigroups $\bar{\Omega}_{A} \mathbf{V}$. 
Lemma 4.5 Let $\mathbf{V}$ be a pseudovariety of semigroups and let $\pi$ be an infinite pseudoword of $\bar{\Omega}_{A} \mathbf{V}$. Then there exist $\pi_{1}, \rho, \pi_{2} \in \bar{\Omega}_{A} \mathbf{V}$ such that $\pi=\pi_{1} \rho^{\omega} \pi_{2}$.

As an easy consequence, we derive the following property of the infinite elements of $\bar{\Omega}_{A} \mathbf{L S l}$.

Corollary 4.6 If $\pi \in \bar{\Omega}_{A} \mathbf{L S I} \backslash A^{+}$, then $\pi^{\omega}=\pi^{2}$.

The next result presents a well-known property of pseudowords over $\mathbf{K}$.

Lemma 4.7 Let $\pi, \rho \in \bar{\Omega}_{A} \mathbf{S}$ be infinite pseudowords such that $p_{\mathbf{K}}(\pi)=p_{\mathbf{K}}(\rho)$. Then there exist factorizations $\pi=\pi_{1} \pi_{2}$ and $\rho=\pi_{1} \rho_{2}$ where $\pi_{1}, \rho_{1}, \rho_{2}$ are infinite. Moreover, if $p_{\mathbf{K}}(\pi)=v u^{+\infty}$ where $v \in A^{*}$ and $u \in A^{+}$, then one can choose $\pi_{1}=v u^{\omega}$.

A dual result holds for $\mathbf{D}$.

\subsection{The $\kappa$-word problem for LSl}

In this subsection we briefly recall the solution of the $\kappa$-word problem for LSl obtained by the first author [14].

A term of rank 0 is a $\kappa$-term not involving the $\omega$-power, that is, is an element of $\Omega_{A} \mathbf{S}\left(=A^{+}\right)$. A term of rank 1 is a $\kappa$-term $\pi$ of the form

$$
\pi=u_{0}^{\prime} u_{1}^{\omega} u_{1}^{\prime} u_{2}^{\omega} \cdots u_{m}^{\omega} u_{m}^{\prime}
$$

with $m \geq 1, u_{0}^{\prime}, \ldots, u_{m}^{\prime} \in A^{*}$ and $u_{1}, \ldots, u_{m} \in A^{+}$. It is clear that $p_{\mathbf{K}}(\pi)$ is the rightinfinite word $u_{0}^{\prime} u_{1}^{+\infty}$ and $p_{\mathbf{D}}(\pi)$ is the left-infinite word $u_{m}^{-\infty} u_{m}^{\prime}$. The $\kappa$-term $\pi$ is said to be in reduced form when: $u_{j}$ is a Lyndon word for $j=1, \ldots, m ; u_{0}^{\prime} u_{1}^{+\infty}$ and $u_{m}^{-\infty} u_{m}^{\prime}$ are words in normal form; $u_{j}^{-\infty} u_{j}^{\prime} u_{j+1}^{+\infty}$ is a non-periodic word for $j=1, \ldots, m-1$. In this case, we denote by $B_{\pi}$ the set of all non-periodic bi-infinite factors of $\pi$, that is,

$$
B_{\pi}=\left\{u_{j}^{-\infty} u_{j}^{\prime} u_{j+1}^{+\infty} \mid j=1, \ldots, m-1\right\} .
$$

Recall that, if a pseudoidentity $\pi=\rho$ holds in LSl, then either $\pi$ and $\rho$ are the same finite word or they both are infinite pseudowords. The following decision criterion to test if two infinite $\kappa$-terms (i.e., $\kappa$-terms of rank at least 1 ) are equal over $\mathbf{L S l}$ is a simple reformulation of [14, Theorem 7.1].

Proposition 4.8 Let $\pi \in \Omega_{A}^{\kappa} \mathbf{S}$ be an infinite $\kappa$-term. Then, there is a rank $1 \kappa$-term $\pi_{1}=u_{0}^{\prime} u_{1}^{\omega} u_{1}^{\prime} u_{2}^{\omega} \cdots u_{m}^{\omega} u_{m}^{\prime}$, in reduced form, such that $\mathbf{L S l} \models \pi=\pi_{1}$.

Moreover, if $\rho \in \Omega_{A}^{\kappa} \mathbf{S}$ is another infinite $\kappa$-term and $\rho_{1}=v_{0}^{\prime} v_{1}^{\omega} v_{1}^{\prime} v_{2}^{\omega} \cdots v_{p}^{\omega} v_{p}^{\prime}$ is a rank $1 \kappa$-term in reduced form such that $\mathbf{L S l}=\rho=\rho_{1}$, then $\mathbf{L S l}$ verifies $\pi=\rho$ if and only if $u_{0}^{\prime} u_{1}^{+\infty}=v_{0}^{\prime} v_{1}^{+\infty}, u_{m}^{-\infty} u_{m}^{\prime}=v_{p}^{-\infty} v_{p}^{\prime}$ and $B_{\pi_{1}}=B_{\rho_{1}}$. Furthermore, it is effectively decidable whether LSl verifies $\pi=\rho$ or not.

Let $\pi \in \bar{\Omega}_{A} \mathbf{S}$ be an infinite pseudoword such that LSl $\models \pi=w$ for some $\kappa$-term $w$. As a consequence of Proposition 4.8, we may define $B_{\pi}$ as being the set $B_{\pi_{1}}$, where $\pi_{1}$ is any rank $1 \kappa$-term in reduced form such that $\mathbf{L S I} \models \pi=\pi_{1}$.

\section{Some combinatorial results}

In this section, we briefly recall some definitions on words and some results of 15, Section 4] that will be used latter. 


\subsection{Centers of bi-infinite words}

Let $w \in A^{+}$and let $u=w[l, r]$ be an occurrence of a factor $u$ in $w$. An occurrence $v=w\left[l, r^{\prime}\right]$, with $r \leq r^{\prime}$, of a factor $v$ in $w$ is said to be a right-extension of the occurrence $w[l, r]$. In this case, the word $v$ itself is said to be a right-extension (in $w$ ) of the occurrence $w[l, r]$.

Definition 5.1 (allowed occurrence) Let $u \in A^{+}$and let $\vec{u} \in A^{+}$be a right-extension of $u$. We say that an occurrence $u=w[l, r]$ in a word $w \in A^{+}$is allowed in $w$ relative to $\vec{u}$ if $\vec{u}$ is a right-extension in $w$ of the occurrence $w[l, r]$.

For instance, let $u=a b a$ and let $\vec{u}=a b a a b c$. Then $w=c a b \mathbf{a b a} a b c a a b a \mathbf{a b a} a b c$ has two allowed occurrences of $u: w[4,6]$ and $w[14,16]$; and two occurrences of $u$ not allowed: $w[2,4]$ and $w[11,13]$. If $\vec{u}=a b a b b$ then $u$ has no allowed occurrences in $w$.

Definition 5.2 (centers of a bi-infinite word) Let $\mathbf{w} \in A^{\mathbb{Z}}$ be a bi-infinite word. For every pair of integers $p, q \in \mathbb{N}_{0}$, the factor $\mathbf{w}[-p, q]$ is said to be a center of $\mathbf{w}$.

The dual (for left-extensions) of the following result was proved in [15, Lemma 4.2].

Lemma 5.3 Let $B=\left\{\mathbf{w}_{1}, \ldots, \mathbf{w}_{n}\right\}$ be a finite set of non-periodic bi-infinite words such that $\mathbf{w}_{i} \nsim \mathbf{w}_{j}$ for every $i, j \in\{1, \ldots, n\}$ with $i \neq j$. For each $\ell \in\{1, \ldots, n\}$ let also $c_{\ell}=\mathbf{w}_{\ell}\left[-p_{\ell}, q_{\ell}\right]$ be a center of $\mathbf{w}_{\ell}$ with $p_{\ell} \geq Q$ for a fixed $Q$ (depending on $B$ ) chosen sufficiently large.

For each $\ell$ there is a center $\overrightarrow{c_{\ell}}=\mathbf{w}_{\ell}\left[-p_{\ell}, q_{\ell}^{\prime}\right]$ of $\mathbf{w}_{\ell}$ with $q_{\ell} \leq q_{\ell}^{\prime}$ (so that $\overrightarrow{c_{\ell}}$ is a rightextension of $c_{\ell}$ ) such that the following property is verified, for every $i, j \in\{1, \ldots, n\}$ (where $i$ and $j$ may be the same):

any two distinct occurrences of $c_{i}$ and $c_{j}$ in a finite word $w \in A^{+}$, which are allowed relative to $\overrightarrow{c_{i}}$ and $\overrightarrow{c_{j}}$ respectively, are disjoint.

This property (5.1), of allowed occurrences of certain centers of bi-infinite words being necessarily disjoint, is essential to our purposes. Indeed, in Section 6, we will need to transform occurrences of certain factors (which are centers of bi-infinite words) in a word and, so, we need these occurrences to be disjoint. The definition of allowed occurrences in a word was introduced with this purpose. We will transform only allowed occurrences of these centers.

\subsection{Marked factors}

In what follows we recall some notions, and their basic properties, introduced in [15]. We will also fix several integers already used in that paper. Let us begin by recalling some of them.

Definition 5.4 (constants $k, k^{\prime}$ and $\left.k^{\prime \prime}\right)$ We let:

- $k$ represent the number $|S|$ of elements of a finite A-generated semigroup $S$. We assume for the rest of the paper that $S$ and $k$ are fixed. 
- $k^{\prime}=6 k|A|^{3 k}$, and notice that this constant is large enough to guarantee that, if $u_{1}, \ldots, u_{r}$ are all the factors of a word $w \in A^{+}$with $2 k \leq\left|u_{i}\right|<3 k$ and if $\operatorname{docc}\left(u_{i}, w\right)>$ $k^{\prime}-2$ for every $1 \leq i \leq r$, then it is possible to choose one occurrence of each factor $u_{i}$ such that these occurrences are pairwise disjoint. We will be interested in these factors $u_{i}$ because they can be factored in the form $u_{i}=u_{i, 1} u_{i, 2} u_{i, 3}$ with $\left|u_{i, 1}\right|=\left|u_{i, 3}\right|=k$ and $\left|u_{i, 2}\right|<k$.

- $k^{\prime \prime}=\left[2 k^{\prime}(3 k-1)\right]^{|A|^{3 k-1}+1}$, and observe that the definition of $k^{\prime \prime}$ is motivated by Lemma 5.6 below.

A finite word $v$ is said to be $k^{\prime}$-abundant if $\operatorname{docc}(y, v) \geq k^{\prime}$ for all factors $y$ of $v$ with length $3 k-1$.

Let $w=a_{1} a_{2} \cdots a_{n}\left(a_{i} \in A\right)$ be a finite word, with $n \geq 3 k-1$. A $k^{\prime \prime}$-neighborhood of an occurrence $u=w[i, j]$ of a factor $u$ in $w$ is an occurrence $v=w\left[i^{\prime}, j^{\prime}\right]$ extending $w[i, j]$ (i.e., such that $i^{\prime} \leq i$ and $j^{\prime} \geq j$ ) and such that $|v| \leq k^{\prime \prime}$. An occurrence $u=w[i, j]$ of a factor $u$ of length $3 k-1$ in $w$ will be said to be free if there exists a $k^{\prime \prime}$-neighborhood $v$ of $w[i, j]$ such that $v$ is $k^{\prime}$-abundant. Notice that, in this case, every occurrence of a factor $y$, of length $3 k-1$, in the $k^{\prime \prime}$-neighborhood $v$ is free. The occurrence $u=w[i, j]$ and the letters $a_{i}, a_{i+1}, \ldots, a_{j}$ will be said to be marked if $w[i, j]$ is not free.

The next lemma follows easily from the above definitions.

Lemma 5.5 There is a unique factorization

$$
w=w_{0} v_{1} w_{1} v_{2} \cdots v_{q} w_{q}
$$

such that

- $q \geq 0$;

- $w_{0}, w_{q} \in A^{*}, w_{1}, \ldots, w_{q-1}, v_{1}, \ldots, v_{q} \in A^{+}$;

- for each $1 \leq i \leq q$, the letters of $v_{i}$ are marked;

- for each $0 \leq j \leq q$, the letters of $w_{j}$ are not marked.

This factorization is called the marked factorization of $w$ (for $k$ ). The factors $v_{1}, \ldots, v_{q}$ (resp. $w_{0}, \ldots, w_{q}$ ) are said to be the marked factors (resp. the free factors) of $w$ (for $k$ ).

The above process of marking letters of a given word $w \in A^{+}$is a way to identify the factors of a given length $3 k-1$ that (locally) have "few" occurrences: the definition of what "few" means is made in such a way that the marked factors have bounded lengths, as shown by the following lemma. On the contrary the free factors are "big" and have no bounds on its lengths.

Lemma 5.6 Let $w=w_{0} v_{1} w_{1} v_{2} \cdots v_{q} w_{q}$ be the marked factorization of a word $w \in A^{+}$ of length at least $3 k-1$. If $q \geq 1$, then $3 k-1 \leq\left|v_{i}\right|<k^{\prime \prime}$ for every $1 \leq i \leq q$. 


\section{Complete $\kappa$-reducibility of LSl}

In this section, we prove the main result of the paper.

Theorem 6.1 The pseudovariety LSI is completely $\kappa$-reducible.

The proof is spread over subsections 6.1 to 6.6. Since the $\kappa$-word problem for LSl is decidable by Proposition 4.8, the complete tameness of $\mathbf{L S l}$ follows immediately from Theorem 6.1.

Theorem 6.2 The pseudovariety $\mathbf{L S l}$ is completely $\kappa$-tame.

A decidability consequence of complete tameness of $\mathbf{L S l}$ is presented in Theorem 6.3 below, a result which follows from [21, Chapter 3]. The reader is referred to this book for details and missing definitions. For a pseudovariety $\mathrm{R}$ of relational morphisms and a pseudovariety $\mathbf{V}$ of semigroups, denote by $\operatorname{Rq}(\mathbf{V})$ the following pseudovariety of semigroups,

$$
\mathbf{R q}(\mathbf{V})=\{S \in \mathbf{S}: \text { there exists } \theta \in \mathbf{R} \text { and } T \in \mathbf{V} \text { with } \theta: S \rightarrow T\} .
$$

Theorem 6.3 The pseudovariety $\mathrm{Rq}(\mathbf{L S l})$ is decidable whenever $\mathrm{R}$ is a pseudovariety of relational morphisms with a finite basis of pseudoidentities of the form $(A, u=v, \mathcal{S})$ with $\mathcal{S}$ being a finite system of $\kappa$-equations.

\subsection{Initial considerations}

Suppose that we are given a finite system $\mathcal{S}$ of $\kappa$-equations and a solution $\delta$ of $\mathcal{S}$ over LSl with respect to a triple $(\varphi, \gamma, \psi)$. To prove the complete $\kappa$-reducibility of $\mathbf{L S l}$, we need to construct a $\kappa$-solution $\delta^{\prime}$ of $\mathcal{S}$ over $\mathbf{L S l}$ with respect to $(\varphi, \gamma, \psi)$. As we observe below, the liberty to choose the $\delta^{\prime}$ label of some variables is very restricted.

Obs. 1. Suppose that $x \in X$ is a variable such that $\delta(x)=u$ with $u \in A^{+}$. By definition of complete $\kappa$-reducibility, we must have $\psi \circ \delta=\gamma=\psi \circ \delta^{\prime}$. Thus, in particular, $\psi\left(\delta^{\prime}(x)\right)=\psi(u)$. Since we are given a morphism $\psi: \bar{\Omega}_{A} \mathbf{S} \rightarrow S$ into a fixed (but arbitrary) finite semigroup $S$, it is possible that the equality $\psi\left(\delta^{\prime}(x)\right)=\psi(u)$ holds only when $\delta^{\prime}(x)=u$. This is the case, for instance, when $u=a$ and $S=\{a, 0\}$ is the monogenic semigroup generated by $a$, defined by the relation $a^{2}=0$. Therefore, in that case we would not have any choice; we would be obliged to define $\delta^{\prime}(x)=u$. However, since we want to define an algorithm to construct $\delta^{\prime}$ that should work with any given system and solution, we thus "need" to define $\delta^{\prime}(x)=u=\delta(x)$ in general.

Obs. 2. Now, suppose that $x \in X$ is such that LSl $=\delta(x)=u^{\omega}$ with $u \in A^{+}$. Since $\mathcal{S}$ is an arbitrary system of $\kappa$-terms, it could include, for instance, the equation $x=y^{\omega}$ with $y$ a variable such that $\delta(y)=u$, because in that case $\mathbf{L S l} \models \delta(x)=\delta(y)^{\omega}$. As LSl $=\delta^{\prime}(x)=\delta^{\prime}(y)^{\omega}$, and we "must have" $\delta^{\prime}(y)=u$ by Obs. 1, we thus "need" to choose for $\delta^{\prime}(x)$ a $\kappa$-term such that $\mathbf{L S l} \models \delta^{\prime}(x)=u^{\omega}=\delta(x)$.

More generally, suppose that $\delta(x)$ is given by a non-explicit $\kappa$-term when projected into $\bar{\Omega}_{A} \mathbf{L S l}$ (see Example 6.17 for a case in which $\delta(x)$ is not itself a $\kappa$-term). By Proposition 4.8 this is equivalent to the existence of a rank $1 \kappa$-term $w=v_{0} u_{1}^{\omega} v_{1} u_{2}^{\omega} \cdots u_{n}^{\omega} v_{n}$ in reduced form such that $\mathbf{L S l} \models \delta(x)=w$. Therefore, by similarity with the situation above, it is not difficult to deduce that we should have $\mathbf{L S l}=\delta^{\prime}(x)=w=\delta(x)$. 
Obs. 3. Suppose at last that $x \in X$ is such that $\mathbf{K} \models \delta(x)=v u^{\omega}$ with $v \in A^{*}$ and $u \in A^{+}$. Then, $\delta(x)=v \pi$ for some pseudoword $\pi$ such that $\mathbf{K}=\pi=u^{\omega}$. Then, by Proposition 4.1, its clear that LSI $=\pi=u \pi$, and we may assume that $x=y z$ and $z=t z$ are equations of $\mathcal{S}$ with $\delta(y)=v, \delta(z)=\pi$ and $\delta(t)=u$. Hence, LSl must verify $\delta^{\prime}(x)=\delta^{\prime}(y) \delta^{\prime}(z)$ and $\delta^{\prime}(z)=\delta^{\prime}(t) \delta^{\prime}(z)=u \delta^{\prime}(z)$, which implies that $\mathbf{K}$ verifies $\delta^{\prime}(z)=u^{\omega}$ and so also $\delta^{\prime}(x)=\delta^{\prime}(y) \delta^{\prime}(z)=v u^{\omega}=\delta(x)$.

Dually, if $x \in X$ is such that $\mathbf{D} \models \delta(x)=u^{\omega} v$ with $v \in A^{*}$ and $u \in A^{+}$, then we should have $\mathbf{D} \models \delta^{\prime}(x)=u^{\omega} v=\delta(x)$.

Observations 1 and 2 above suggest that, for a variable $x$ labeled under $\delta$ by a $\kappa$ term when projected into $\bar{\Omega}_{A} \mathbf{L S l}$, we should choose for $\delta^{\prime}(x)$ a $\kappa$-term verifying $\mathbf{L S} \mathbf{l} \models$ $\delta^{\prime}(x)=\delta(x)$. On the other hand, Observation 3 suggests that, if $x$ is a variable such that the projection of $\delta(x)$ into $\bar{\Omega}_{A} \mathbf{K}$ (resp. into $\bar{\Omega}_{A} \mathbf{D}$ ) is an ultimately periodic word $v u^{+\infty}$ (resp. $u^{-\infty} v$ ), then we should choose for $\delta^{\prime}(x)$ a $\kappa$-term whose projection into $\bar{\Omega}_{A} \mathbf{K}$ (resp. into $\left.\bar{\Omega}_{A} \mathbf{D}\right)$ is also $v u^{+\infty}\left(\right.$ resp. $\left.u^{-\infty} v\right)$. The $\kappa$-solution $\delta^{\prime}$, to be defined in Subsection 6.5 below, will respect these constraints.

\subsection{Simplifications on the system of equations}

Let us proceed with the simplifications which are the objective of this subsection. By the following result, proved in [8, Proposition 3.1], we may restrict the problem to consider only systems without parameters.

Proposition 6.4 Let $\mathbf{V}$ be an arbitrary pseudovariety and let $\sigma$ be any implicit signature. If $\mathbf{V}$ is $\sigma$-reducible for systems of equations of $\sigma$-terms without parameters, then $\mathbf{V}$ is completely $\sigma$-reducible.

For the rest of the paper, we will work only with the pseudovariety LSl and all the solutions that we consider are over LSI. So, by Proposition 6.4, it suffices to consider a finite system $\mathcal{S}$ of $\kappa$-equations

$$
u_{i}=v_{i} \quad(i=1, \ldots, h),
$$

with $u_{i}, v_{i} \in \Omega_{X}^{\kappa} \mathbf{S}$. Consider also a solution $\delta$ of $\mathcal{S}$ over $\mathbf{L S l}$ with respect to a pair $(\gamma, \psi)$.

Before describing new simplifications on the system and the solution, we illustrate them with an example.

Example 6.5 Suppose that $X=\{x, y, z, w, t\}$ is the set of variables and that $\mathcal{S}$ has the unique equation of $\kappa$-terms of rank $\leq 2$

$$
\left(x^{\omega} y\right)^{\omega} z=(w t)^{\omega} z .
$$

Since $\delta$ is a solution over $\mathbf{L S 1}$, it follows that $\mathbf{L S l}$ verifies

$$
\delta\left(\left(x^{\omega} y\right)^{\omega} z\right)=\delta\left((w t)^{\omega} z\right) .
$$

As $\delta$ is a $\kappa$-morphism and so, in particular, commutes with $\omega$-powers, we deduce that LSl satisfies

$$
\left(\delta(x)^{\omega} \delta(y)\right)^{\omega} \delta(z)=(\delta(w) \delta(t))^{\omega} \delta(z) .
$$


Now, applying Corollary 4.6, we obtain

$$
\mathbf{L S} 1 \models \delta(x)^{\omega} \delta(y) \delta(x)^{\omega} \delta(y) \delta(z)=(\delta(w) \delta(t))^{\omega} \delta(z) .
$$

Then, $\delta$ is a solution over $\mathbf{L S l}$ with respect to $(\gamma, \psi)$ of the system $\mathcal{S}_{0}$ with the single equation of $\kappa$-terms of rank $\leq 1$

$$
x^{\omega} y x^{\omega} y z=(w t)^{\omega} z .
$$

We suppose furthermore that $\delta(w)$ and $\delta(t)$ are finite pseudowords and that $\delta(x)$ is an infinite pseudoword. We consider a new variable $v$ and a new equation $v=w t$ and define mappings $\delta_{1}$ and $\gamma_{1}$ extending $\delta$ and $\gamma$ to $X_{1}=X \cup\{v\}$ by defining $\delta_{1}(v)=\delta(w) \delta(t)$ and $\gamma_{1}(v)=\psi \circ \delta_{1}(v)$. Therefore, again by Corollary 4.6, LSl verifies

$$
\delta_{1}(x) \delta_{1}(x) \delta_{1}(y) \delta_{1}(x) \delta_{1}(x) \delta_{1}(y) \delta_{1}(z)=\delta_{1}(v)^{\omega} \delta_{1}(z) .
$$

Then, $\delta_{1}$ is a solution over $\mathbf{L S l}$ with respect to $\left(\gamma_{1}, \psi\right)$ of the following system $\mathcal{S}_{1}$

$$
\left\{\begin{array}{l}
x x y x x y z=v^{\omega} z \\
v=w t .
\end{array}\right.
$$

Suppose that $\mathcal{S}_{1}$ admits a $\kappa$-solution $\delta_{1}^{\prime}$ over $\mathbf{L S l}$ with respect to $\left(\gamma_{1}, \psi\right)$ such that $\delta_{1}^{\prime}(x)$ is infinite. [We will prove in Subsection 6.5 that such a $\kappa$-solution exists for such type of equations.] Then $\delta^{\prime}=\left.\delta_{1}^{\prime}\right|_{X}$ is a $\kappa$-solution of $\mathcal{S}$ over $\mathbf{L S l}$ with respect to $(\gamma, \psi)$.

Motivated by this example, we will consider finite systems of $\kappa$-equations

$$
u_{i, 1} \cdots u_{i, p_{i}}=u_{i, p_{i}+1} \cdots u_{i, q_{i}} \quad(i=1, \ldots, h),
$$

with solutions $\delta$ over $\mathbf{L S l}$ with respect to a pair $(\gamma, \psi)$ where, for each $j \in\left\{1, \ldots, q_{i}\right\}$, $u_{i, j}$ is either a variable or $u_{i, j}=x_{i, j}^{\omega}$ with $x_{i, j}$ a variable such that $\delta\left(x_{i, j}\right) \in A^{+}$.

Example 6.5 (namely the property required by the $\kappa$-solution $\delta_{1}^{\prime}$ ) also motivates the following definition.

Definition 6.6 (FT $\kappa$-reducibility) We will say that LSl is FT ("first type") $\kappa$-reducible if, for each system $\mathcal{S}$ and solution $\delta$ of form (6.2) there exists a $\kappa$-solution $\delta^{\prime}$ over LSl such that, for each $x \in X, \delta^{\prime}(x)$ is infinite when $\delta(x)$ is infinite.

Now we show that the complete $\kappa$-reducibility of LSl is a consequence of its FT $\kappa$-reducibility.

Proposition 6.7 If LSl is FT $\kappa$-reducible, then LSl is completely $\kappa$-reducible.

Proof. By Proposition 6.4, it suffices to consider a finite system $\mathcal{S}$ of type (6.1). Let $\delta$ be a solution of $\mathcal{S}$ over $\mathbf{L S}$ with respect to a pair $(\gamma, \psi)$, and let us apply the process illustrated in Example 6.5.

We choose any subterm of the form $u^{\omega}$, with $u$ an infinite $\kappa$-term, and we replace $u^{\omega}$ by $u^{2}$. We repeat the same step in the new system thus obtained, and we iterate this process until possible. Since the rank of the subterms replaced decrease, this process must stop with a system $\mathcal{S}_{0}$ of equations of $\kappa$-terms of rank $\leq 1$. Notice that $\mathcal{S}_{0}$ is 
unique, that is, $\delta_{0}$ does not dependent on the order of the substitutions. Moreover, $\delta$ is a solution of $\mathcal{S}_{0}$ over $\mathbf{L S}$ with respect to $(\gamma, \psi)$.

Now, we consider each subterm, of the equations of $\mathcal{S}_{0}$, of the form $u^{\omega}$ (with $u \in X^{+}$ of course). If $\delta(u)$ is an infinite pseudoword, then we replace $u^{\omega}$ by $u^{2}$. Otherwise $\delta(u)$ is a finite word. In this case we add a new variable $v_{u}$ to the set of variables, we add a new equation $v_{u}=u$ to the system of equations, and we extend $\delta$ and $\gamma$ to new mappings $\delta_{1}$ and $\gamma_{1}$ by letting $\delta_{1}\left(v_{u}\right)=\delta(u)$ and $\gamma_{1}\left(v_{u}\right)=\psi \circ \delta_{1}\left(v_{u}\right)$. We obtain a finite system $\mathcal{S}_{1}$ of form (6.2) having $\delta_{1}$ as a solution over LSl with respect to $\left(\gamma_{1}, \psi\right)$. By hypothesis there exists a $\kappa$-solution $\delta_{1}^{\prime}$ of $\mathcal{S}_{1}$ over $\mathbf{L S l}$ with respect to $\left(\gamma_{1}, \psi\right)$ such that $\delta_{1}^{\prime}(x)$ is infinite when $\delta_{1}(x)$ is infinite. Therefore $\delta^{\prime}=\left.\delta_{1}^{\prime}\right|_{X}$ is a $\kappa$-solution of $\mathcal{S}$ over LSl with respect to $(\gamma, \psi)$.

We proceed with our next reduction on the problem. We consider a system of word equations, that is a system $\mathcal{S}$ of equations of the form

$$
x_{i, 1} \cdots x_{i, p_{i}}=x_{i, p_{i}+1} \cdots x_{i, q_{i}} \quad(i=1, \ldots, h)
$$

where $x_{i, j}$ is a variable for all $j \in\left\{1, \ldots, q_{i}\right\}$, and let $\delta$ be a solution of $\mathcal{S}$ over $\mathbf{L S l}$ with respect to a pair $(\gamma, \psi)$, labeling each variable by an infinite pseudoword.

Definition 6.8 (ST $\kappa$-reducibility) We will say that LSl is ST ("second type") $\kappa$ reducible if, for every system $\mathcal{S}$ and solution $\delta$ of form (6.3) and every integer $M \geq 1$, there exists a $\kappa$-solution $\delta^{\prime}=\delta^{\prime}(\mathcal{S}, \delta, M)$ over $\mathbf{L S l}$ with respect to $(\gamma, \psi)$ such that for each variable $x \in X$,

(ST.1) $\delta^{\prime}(x)$ is infinite;

(ST.2) if $\mathbf{L S l} \models \delta(x)=v u^{\omega}$, where $u \neq 1$ and $v$ are finite words, then $\mathbf{L S l} \models \delta^{\prime}(x)=$ $\delta(x)$;

(ST.3) if $\delta(x)=u \pi$, where $u \in A^{*}$ with $|u| \leq M$ and $\pi \in \bar{\Omega}_{A} \mathbf{S}$, then $\delta^{\prime}(x)=u \pi^{\prime}$ where $\pi^{\prime} \in \Omega_{A}^{\kappa} \mathbf{S}$ is such that $\psi(\pi)=\psi\left(\pi^{\prime}\right)$.

Moreover, if $y$ is another variable, $v \in A^{*}$ with $|v| \leq M$ and $\rho \in \bar{\Omega}_{A} \mathbf{S}$ are such that $\delta(y)=v \rho$ and $\mathbf{L S} 1=\pi=\rho$, then $\delta^{\prime}(y)=v \rho^{\prime}$ where $\rho^{\prime} \in \Omega_{A}^{\kappa} \mathbf{S}$ is such that $\psi(\rho)=\psi\left(\rho^{\prime}\right)$ and $\mathbf{L S} 1 \models \pi^{\prime}=\rho^{\prime}$. In particular, if $\mathbf{L S l} \models \delta(x)=\delta(y)$, then $\mathbf{L S l} \models \delta^{\prime}(x)=\delta^{\prime}(y)$.

As one notices by this definition, to permit the simplification of the system and the solution, we need to require more complex properties to be preserved. On the other hand, although LS1 is an autodual pseudovariety, conditions (ST.2). (ST.3) above are not symmetric. This happens because (cf. the proof of Proposition 6.9 below) in order to reduce the problem to a system $\mathcal{S}$ and solution $\delta$ of form (6.3), when a general system and solution are given we "absorb" the variables which are labeled by finite pseudowords into the variables to their right.

Proposition 6.9 If LSl is ST $\kappa$-reducible, then LSl is completely $\kappa$-reducible.

Proof. By Proposition 6.7, it suffices to show that $S T \kappa$-reducibility implies $F T \kappa$ reducibility of LSl. So, let $\mathcal{S}$ be a finite system and let $\delta$ be a solution of $\mathcal{S}$ over LSl with respect to a pair $(\gamma, \psi)$, of type (6.2). That is $\mathcal{S}$ is a system of equations

$$
u_{i, 1} \cdots u_{i, p_{i}}=u_{i, p_{i}+1} \cdots u_{i, q_{i}} \quad(i=1, \ldots, h)
$$


where $u_{i, j}$ is either a variable or $u_{i, j}=x_{i, j}^{\omega}$ with $x_{i, j}$ a variable such that $\delta\left(x_{i, j}\right) \in A^{+}$.

We first reduce to the case in which the last factors $u_{i, p_{i}}$ and $u_{i, q_{i}}$ of each equation $i$ are labelled under $\delta$ by infinite pseudowords. That is, we assume that for such systems and solutions there exists a $\kappa$-solution, and prove that a system $\mathcal{S}$ and solution $\delta$ without this extra condition also yields a $\kappa$-solution. We consider an enlarged alphabet $B=A \uplus\{b\}$, an extended set of variables $Y=X \uplus\{\#\}$ and a new system $\mathcal{S}_{1}$ with equations

$$
u_{i, 1} \cdots u_{i, p_{i}} \#=u_{i, p_{i}+1} \cdots u_{i, q_{i}} \# \quad(i=1, \ldots, h)
$$

obtained from the equations of $\mathcal{S}$ simply by the multiplication of both sides of the equation by \#. As pointed out in Subsection $2.2, \bar{\Omega}_{A} \mathbf{S}$ can be viewed as a subsemigroup of $\bar{\Omega}_{B} \mathbf{S}$. So, we let $\delta_{1}$ be the extension of $\delta$ to $Y$ such that $\delta_{1}(\#)=b^{\omega}$. Now, we consider the finite semigroup $S_{1}=S^{1} \times \mathcal{P}(B)$, where $\mathcal{P}(B)=\bar{\Omega}_{B}$ Sl is the power set of $B$. Let $\psi_{1}: \bar{\Omega}_{B} \mathbf{S} \rightarrow S_{1}$ be the unique continuous morphism such that $\psi_{1}(b)=(1,\{b\})$ and $\psi_{1}(a)=(\psi(a),\{a\})$ for each $a \in A$, and let $\gamma_{1}: Y \rightarrow S_{1}$ be defined by $\gamma_{1}=\psi_{1} \circ \delta_{1}$. Since $\delta$ is a solution of $\mathcal{S}$ over LSl with respect to $(\gamma, \psi)$, it is clear that $\delta_{1}$ is a solution of $\mathcal{S}_{1}$ over $\mathbf{L S l}$ with respect to $\left(\gamma_{1}, \psi_{1}\right)$. By assumption there exists a $\kappa$-solution $\delta_{1}^{\prime}$ of $\mathcal{S}_{1}$ over $\mathbf{L S l}$ with respect to $\left(\gamma_{1}, \psi_{1}\right)$. Therefore

$$
\begin{aligned}
& \forall i \in\{1, \ldots, h\} \quad \mathbf{L S} \mathbf{l}=\delta_{1}^{\prime}\left(u_{i, 1} \cdots u_{i, p_{i}} \#\right)=\delta_{1}^{\prime}\left(u_{i, p_{i}+1} \cdots u_{i, q_{i}} \#\right), \\
& \psi_{1} \circ \delta_{1}^{\prime}=\gamma_{1}=\psi_{1} \circ \delta_{1} .
\end{aligned}
$$

By the definition of $\psi_{1}$, it follows from (6.5) that

$$
\begin{aligned}
& \psi \circ \delta^{\prime}=\psi \circ \delta=\gamma \\
& c \circ \delta_{1}^{\prime}=c \circ \delta_{1},
\end{aligned}
$$

where $\delta^{\prime}: X \rightarrow \bar{\Omega}_{A} \mathbf{S}$ is the restriction of $\delta_{1}^{\prime}$ to $X$. Notice that $\delta^{\prime}$ is well-defined since, by (6.7), $c\left(\delta_{1}^{\prime}(x)\right) \subseteq A$ for every $x \in X$. Moreover $c\left(\delta_{1}^{\prime}(\#)\right)=c\left(\delta_{1}(\#)\right)=\{b\}$, so that, by (6.4) and Corollary 4.2 ,

$$
\forall i \in\{1, \ldots, h\} \quad \mathbf{L S} \mathbf{l}=\delta^{\prime}\left(u_{i, 1} \cdots u_{i, p_{i}}\right)=\delta^{\prime}\left(u_{i, p_{i}+1} \cdots u_{i, q_{i}}\right) .
$$

Since $\psi \circ \delta^{\prime}=\gamma$ by (6.6), we deduce that $\delta^{\prime}$ is a $\kappa$-solution of $\mathcal{S}$ over LSl with respect to $(\gamma, \psi)$, which concludes the first reduction.

So, we assume that the above system $\mathcal{S}$ and solution $\delta$ are such that $\delta\left(u_{i, p_{i}}\right)$ and $\delta\left(u_{i, q_{i}}\right)$ are infinite pseudowords, and show how to transform these data into a finite system $\mathcal{S}_{1}$ and a solution $\delta_{1}$ of $\mathcal{S}_{1}$ over $\mathbf{L S l}$ with respect to a pair $\left(\gamma_{1}, \psi\right)$ of the special form (6.3). The new set of variables, denoted by $X_{1}$, will be an extension of $X$, and $\delta_{1}$ and $\gamma_{1}$ will be extensions of $\delta$ and $\gamma$ to $X_{1}$.

Suppose that some $u_{i, j}$ is labelled under $\delta$ by an infinite pseudoword and that $\ell<j$ is minimal such that $u_{i, \ell}, \ldots, u_{i, j-1}$ are variables labelled under $\delta$ by finite pseudowords. In this case, we let $y_{i, j}$ be a new variable, substitute the subterm

$$
u_{i, \ell} \cdots u_{i, j-1} u_{i, j}
$$

by $y_{i, j}$ in the $i$-th equation of $\mathcal{S}$, and define $\delta_{1}\left(y_{i, j}\right)=\delta\left(u_{i, \ell}\right) \cdots \delta\left(u_{i, j-1}\right) \delta\left(u_{i, j}\right)$ and

$$
\gamma_{1}\left(y_{i, j}\right)=\left\{\begin{array}{l}
\gamma\left(u_{i, \ell}\right) \cdots \gamma\left(u_{i, j-1}\right) \gamma\left(u_{i, j}\right) \text { if } u_{i, j} \text { is a variable } \\
\gamma\left(u_{i, \ell}\right) \cdots \gamma\left(u_{i, j-1}\right) \gamma\left(x_{i, j}\right)^{\omega} \text { if } u_{i, j}=x_{i, j}^{\omega} .
\end{array}\right.
$$


This way, all variables of the form $u_{i, m}$ labelled under $\delta$ by finite pseudowords are substituted. Suppose now that some subterm of the form $u_{i, j}=x_{i, j}^{\omega}$ still remains in the new system. In this case, $\delta\left(x_{i, j}\right)$ is finite and we substitute $u_{i, j}$ by a new variable $z_{i, j}$ and define $\delta_{1}\left(z_{i, j}\right)=\delta\left(x_{i, j}\right)^{\omega}$ and $\gamma_{1}\left(z_{i, j}\right)=\gamma\left(x_{i, j}\right)^{\omega}$. After all these substitutions are made, the process of construction of $\mathcal{S}_{1}$ is concluded. Moreover $\delta_{1}$ is clearly a solution of $\mathcal{S}_{1}$ over $\mathbf{L S} \mathbf{l}$ with respect to $\left(\gamma_{1}, \psi\right)$.

Fix an integer

$$
M>\prod_{\substack{1 \leq i \leq h, 1 \leq m \leq q_{i} \\ \delta\left(u_{i, m}\right) \in A^{+}}}\left|\delta\left(u_{i, m}\right)\right| .
$$

By hypothesis LSl is $S T \kappa$-reducible. Therefore, there exists a $\kappa$-solution $\delta_{1}^{\prime}=$ $\delta_{1}^{\prime}\left(\mathcal{S}_{1}, \delta_{1}, M\right)$ of $\mathcal{S}_{1}$ over $\mathbf{L S l}$ with respect to $\left(\gamma_{1}, \psi\right)$ satisfying conditions (ST.1) to (ST.3), We use $\delta_{1}^{\prime}$ to construct a $\kappa$-solution $\delta^{\prime}$, of the original system $\mathcal{S}$, over LSl with respect to $(\gamma, \psi)$ as follows. Let $x \in X$.

- If $\delta(x)$ is finite, then we let $\delta^{\prime}(x)=\delta(x)$.

- If $\delta(x)$ is infinite and $x$ does not occur in some equation of the original system $\mathcal{S}$, then we let $\delta^{\prime}(x)=\delta_{1}^{\prime}(x)$.

- Suppose that $\delta(x)$ is infinite and that $x$ occurs in (some equation of) $\mathcal{S}$. Notice that $x$ may have various occurrences in $\mathcal{S}$. We will see that each such occurrence determines a candidate for $\delta^{\prime}(x)$ and we will prove that all the candidates are equal over LSI, so that any of the candidates can be chosen to be the value of $\delta^{\prime}(x)$.

Let $u_{i, j}$ be an occurrence of $x$. If the subterm $u_{i, j}$ was not replaced in the substitutions that gave origin to system $\mathcal{S}_{1}$, then we let $\pi_{i, j}^{\prime}=\delta_{1}^{\prime}(x)$ be a candidate for $\delta^{\prime}(x)$. Otherwise $u_{i, j}$ (the subterm (6.8) to be more precise) was replaced by the new variable $y_{i, j}$. In this case $\delta_{1}\left(y_{i, j}\right)=u \pi_{i, j}$, where $u=\delta\left(u_{i, \ell}\right) \cdots \delta\left(u_{i, j-1}\right) \in A^{+}$and $\pi_{i, j}=\delta\left(u_{i, j}\right)$. By (6.9), $M>|u|$. Therefore, by condition (ST.3), $\delta_{1}^{\prime}\left(y_{i, j}\right)=u \pi_{i, j}^{\prime}$ where $\pi_{i, j}^{\prime} \in \Omega_{A}^{\kappa} \mathbf{S}$ is such that $\psi\left(\pi_{i, j}\right)=\psi\left(\pi_{i, j}^{\prime}\right)$, and we let $\pi_{i, j}^{\prime}$ be a candidate for $\delta^{\prime}(x)$.

Suppose now that $\pi_{i_{1}, j_{1}}^{\prime}$ and $\pi_{i_{2}, j_{2}}^{\prime}$ are two candidates for $\delta^{\prime}(x)$. It follows immediately from the definition of the candidates and from the second part of condition (ST.3) that $\mathbf{L S l}=\pi_{i_{1}, j_{1}}^{\prime}=\pi_{i_{2}, j_{2}}^{\prime}$. Therefore, we choose for $\delta^{\prime}(x)$ any one of its candidates.

By construction $\delta^{\prime}$ is a $\kappa$-solution of $\mathcal{S}$ over LSl with respect to $(\gamma, \psi)$. Indeed, the equality $\psi \circ \delta^{\prime}=\gamma$ is a simple consequence of $\psi \circ \delta=\gamma$. Moreover, it should be clear from the statements above that to show

$$
\forall i \in\{1, \ldots, h\} \quad \mathbf{L S l} \models \delta^{\prime}\left(u_{i, 1} \cdots u_{i, p_{i}}\right)=\delta^{\prime}\left(u_{i, p_{i}+1} \cdots u_{i, q_{i}}\right)
$$

it suffices to verify that:

(a) $\mathbf{L S l} \models \delta_{1}^{\prime}\left(y_{i, j}\right)=\delta^{\prime}\left(u_{i, \ell} \cdots u_{i, j}\right)$ for every substitution of the form $u_{i, \ell} \cdots u_{i, j} \mapsto y_{i, j}$;

(b) $\mathbf{L S l} \models \delta_{1}^{\prime}\left(z_{i, j}\right)=\delta^{\prime}\left(u_{i, j}\right)$ for every substitution of the form $u_{i, j} \mapsto z_{i, j}$.

The other case being analogous, we only prove this when $u_{i, j}$ is a subterm of the form $x_{i, j}^{\omega}$, and so $x_{i, j} \in X$ is a variable such that $\delta\left(x_{i, j}\right)$ is a finite pseudoword.

For (a), we have $\delta_{1}\left(y_{i, j}\right)=u \delta\left(x_{i, j}\right)^{\omega}$, where $u=\delta\left(u_{i, \ell}\right) \cdots \delta\left(u_{i, j-1}\right) \in A^{+}$. Hence, by condition (ST.2), LSl satisfies $\delta_{1}^{\prime}\left(y_{i, j}\right)=\delta_{1}\left(y_{i, j}\right)$ and so it satisfies also $\delta_{1}^{\prime}\left(y_{i, j}\right)=$ 
$\delta^{\prime}\left(u_{i, \ell} \cdots u_{i, j}\right)$ since, by definition of $\delta^{\prime}, \delta^{\prime}\left(x_{i, j}\right)=\delta\left(x_{i, j}\right)$ and $\delta^{\prime}\left(u_{i, m}\right)=\delta\left(u_{i, m}\right)$ for every $m \in\{\ell, \ldots, j-1\}$.

For (b), we have $\delta_{1}\left(z_{i, j}\right)=\delta\left(x_{i, j}\right)^{\omega}$ and, using again (ST.2), we deduce successively

$$
\mathbf{L S l} \models \delta_{1}^{\prime}\left(z_{i, j}\right)=\delta_{1}\left(z_{i, j}\right)=\delta\left(x_{i, j}\right)^{\omega}=\delta^{\prime}\left(x_{i, j}\right)^{\omega}=\delta^{\prime}\left(u_{i, j}\right) .
$$

Therefore $\delta^{\prime}$ is a $\kappa$-solution of $\mathcal{S}$ over $\mathbf{L S l}$ with respect to $(\gamma, \psi)$. To conclude the proof of the proposition notice that for each variable $x \in X, \delta^{\prime}(x)$ is infinite when $\delta(x)$ is infinite.

To achieve our last reduction on the problem, we need to introduce some notation. Although we will reduce to a more complicated system, this will simplify the description of the solution of the problem. We assume there are given a system and a solution of form (6.3). Notice first that we may as well assume that, if $\mathbf{L S l}$ is ST $\kappa$-reducible, then each variable $x \in X$ has one and only one occurrence in $\mathcal{S}$. Indeed if a variable does not occur in $\mathcal{S}$, we may remove it from the set $X$. On the other hand, if a variable $x$ has more than one occurrence in $\mathcal{S}$, then we may replace each extra occurrence of $x$ by a new variable labelled by $\delta(x)$. If $y$ is one of these new variables and $\delta_{1}^{\prime}$ is a $\kappa$-solution of the new system, then $\mathbf{L S l}=\delta_{1}^{\prime}(x)=\delta_{1}^{\prime}(y)$ by condition (ST.3). Hence, for each variable $x \in X$, we may choose $\delta^{\prime}(x)=\delta_{1}^{\prime}(x)$ to obtain a solution of the original system $\mathcal{S}$.

In the rest of the paper, we will introduce several functions defined on the set of variables. For one such function $f$ and a variable $x \in X$, the value of $x$ under $f$ will be denoted by $f_{x}$. When we are interested in identifying the (unique) place where $x$ occurs in $\mathcal{S}$, say when $x=x_{i, j}$, we will also denote $f_{x}$ by $f_{i, j}$ (and we will freely adopt this double notation).

Definition 6.10 (words $\mathbf{k}_{x}, \mathbf{d}_{x}, \mathbf{w}_{(i, j)}$ and sets $K_{\mathcal{S}}, D_{\mathcal{S}}, W_{\mathcal{S}}$ ) For each variable $x$, we denote by $\mathbf{k}_{x} \in A^{\mathbb{N}}$ and $\mathbf{d}_{x} \in A^{-\mathbb{N}}$ the projections of $\delta(x)$ into $\bar{\Omega}_{A} \mathbf{K}$ and $\bar{\Omega}_{A} \mathbf{D}$, respectively, and let

$$
K_{\mathcal{S}}=\left\{\mathbf{k}_{x} \mid x \in X\right\} \quad \text { and } \quad D_{\mathcal{S}}=\left\{\mathbf{d}_{x} \mid x \in X\right\} .
$$

For each pair of integers $(i, j)$ with $i \in\{1, \ldots, h\}$ and $j \in\left\{2, \ldots, p_{i}, p_{i}+2, \ldots, q_{i}\right\}$, we denote by $\mathbf{w}_{(i, j)}$ the bi-infinite word

$$
\mathbf{w}_{(i, j)}=\mathbf{d}_{i, j-1} \cdot \mathbf{k}_{i, j}
$$

which is determined by the factor $x_{i, j-1} x_{i, j}$ of the $i$-th equation of $\mathcal{S}$ (i.e., $\mathbf{w}_{(i, j)}=$ $\left.\overleftrightarrow{\delta\left(x_{i, j-1}\right) \delta\left(x_{i, j}\right)}\right)$, and let $W_{\mathcal{S}}$ represent the set of all such words $\mathbf{w}_{(i, j)}$

For each $i \in\{1, \ldots, h\}$ we let further

$$
\begin{array}{ll}
K_{i}(0)=\left\{\mathbf{k}_{i, j} \mid j=1, \ldots, p_{i}\right\}, & K_{i}(1)=\left\{\mathbf{k}_{i, j} \mid j=p_{i}+1, \ldots, q_{i}\right\}, \\
D_{i}(0)=\left\{\mathbf{d}_{i, j} \mid j=1, \ldots, p_{i}\right\}, & D_{i}(1)=\left\{\mathbf{d}_{i, j} \mid j=p_{i}+1, \ldots, q_{i}\right\}, \\
W_{i}(0)=\left\{\mathbf{w}_{(i, j)} \mid j=2, \ldots, p_{i}\right\}, & W_{i}(1)=\left\{\mathbf{w}_{(i, j)} \mid j=p_{i}+2, \ldots, q_{i}\right\} .
\end{array}
$$

Notice that $W_{\mathcal{S}}$ may be empty (when each equation is of the type $x_{i, 1}=x_{i, 2}$ ). This case is easier and it could be treated separately. However, to avoid having to consider two cases, we may assume that this set is non-empty since we could, for instance, proceed as in the proof of Proposition 6.9 and multiply both sides of one equation of $\mathcal{S}$ by a new variable \#. 
We say that two right-infinite words $w_{1}, w_{2} \in A^{\mathbb{N}}$ are confinal if they have a common suffix $v \in A^{\mathbb{N}}$, that is, if $w_{1}=u_{1} v$ and $w_{2}=u_{2} v$ for some words $u_{1}, u_{2} \in A^{*}$. As one easily verifies, the relation $\theta_{1}$ defined, for each $\mathbf{k}_{x}, \mathbf{k}_{y} \in K_{\mathcal{S}}$, by

$$
\mathbf{k}_{x} \theta_{1} \mathbf{k}_{y} \quad \text { if and only if } \mathbf{k}_{x} \text { and } \mathbf{k}_{y} \text { are confinal }
$$

is an equivalence on $K_{\mathcal{S}}$. The notion of left-infinite confinal words can be introduced symmetrically. An equivalence $\theta_{0}$ on $D_{\mathcal{S}}$ can also be defined by symmetry.

Notice that, since $\delta$ is a solution of $\mathcal{S}$ both over $\mathbf{K}$ and over $\mathbf{D}$, we have the equalities

$$
\mathbf{k}_{i, 1}=\mathbf{k}_{i, p_{i}+1} \text { and } \mathbf{d}_{i, p_{i}}=\mathbf{d}_{i, q_{i}} \text { for all } i \in\{1, \ldots, h\} .
$$

Definition 6.11 (synchronized system) We say that the pair $(\mathcal{S}, \delta)$ is synchronized if the following conditions are verified:

(Sy.1) If $\mathbf{d}_{x}$ and $\mathbf{d}_{y}$ are two confinal words of $D_{\mathcal{S}}$, then $\mathbf{d}_{x}=\mathbf{d}_{y}$. Moreover, if $\mathbf{d}_{x}$ is ultimately periodic, then $\mathbf{d}_{x}=u^{-\infty}$ where $u$ is a Lyndon word;

(Sy.2) If $\mathbf{w}_{(i, j)}, \mathbf{w}_{(\ell, m)} \in W_{\mathcal{S}}$ are such that $\mathbf{w}_{(i, j)} \sim \mathbf{w}_{(\ell, m)}$, then $\mathbf{w}_{(i, j)}=\mathbf{w}_{(\ell, m)}$;

(Sy.3) $W_{i}(0)=W_{i}(1)$ for every $i$. Therefore, by (6.10), also $K_{i}(0)=K_{i}(1)$ and $D_{i}(0)=$ $D_{i}(1)$;

(Sy.4) $W_{i}(0)$ contains the set $B_{\delta\left(x_{i, j}\right)}$ of non-periodic bi-infinite factors of $\delta\left(x_{i, j}\right)$, for every variable $x_{i, j}$ such that $\delta\left(x_{i, j}\right)$ is a $\kappa$-term when projected into $\bar{\Omega}_{A} \mathbf{L S l}$;

(Sy.5) $W_{\mathcal{S}}$ does not contain periodic words.

When the solution is understood, we will simply say that system $\mathcal{S}$ is synchronized.

Definition 6.12 (TT $\kappa$-reducibility) We will say that LSl is TT ("third type") $\kappa$ reducible if, for every synchronized pair $(\mathcal{S}, \delta)$ and every integer $M \geq 1$, there exists a $\kappa$-solution $\delta^{\prime}=\delta^{\prime}(\mathcal{S}, \delta, M)$ over LSl with respect to $(\gamma, \psi)$ such that for each variable $x \in X$,

(TT.1) $\delta^{\prime}(x)$ is infinite;

(TT.2) if $\mathbf{K} \models \delta(x)=v u^{\omega}$, where $u \neq 1$ and $v$ are finite words, then $\mathbf{K} \models \delta^{\prime}(x)=\delta(x)$;

(TT.3) if $\mathbf{D} \models \delta(x)=u^{\omega}$, where $u \neq 1$ is a finite word, then $\mathbf{D} \models \delta^{\prime}(x)=\delta(x)$;

(TT.4) if $\mathbf{L S l} \models \delta(x)=w$ for some $\kappa$-term $w$, then $\mathbf{L S l} \models \delta^{\prime}(x)=\delta(x)$;

(TT.5) if $\delta(x)=u \pi$, where $u \in A^{*}$ with $|u| \leq M$ and $\pi \in \bar{\Omega}_{A} \mathbf{S}$, then $\delta^{\prime}(x)=u \pi^{\prime}$ where $\pi^{\prime} \in \Omega_{A}^{\kappa} \mathbf{S}$ is such that $\psi(\pi)=\psi\left(\pi^{\prime}\right)$.

Moreover, if $y$ is another variable, $v \in A^{*}$ with $|v| \leq M$ and $\rho \in \bar{\Omega}_{A} \mathbf{S}$ are such that $\delta(y)=v \rho$ and $\mathbf{L S}=\pi=\rho$, then $\delta^{\prime}(y)=v \rho^{\prime}$ where $\rho^{\prime} \in \Omega_{A}^{\kappa} \mathbf{S}$ is such that $\psi(\rho)=\psi\left(\rho^{\prime}\right)$ and $\mathbf{L S} \mathbf{l}=\pi^{\prime}=\rho^{\prime}$. In particular, if $\mathbf{L S} \mathbf{l}=\delta(x)=\delta(y)$, then LSl $=\delta^{\prime}(x)=\delta^{\prime}(y)$.

We will prove in the remaining subsections that LSl is TT $\kappa$-reducible. For now, we show that indeed the TT $\kappa$-reducibility of LSl implies the complete $\kappa$-reducibility of LSl. 
Proposition 6.13 If LSl is TT $\kappa$-reducible, then LSl is completely $\kappa$-reducible.

Proof. By Proposition 6.9, it suffices to show that $T T \kappa$-reducibility implies $S T \kappa$ reducibility of $\mathbf{L S l}$. So, let $\mathcal{S}$ be a finite system, let $\delta$ be a solution of $\mathcal{S}$ over LSl with respect to a pair $(\gamma, \psi)$ of type (6.3), and let $M \geq 1$ be an integer. We show that we can reduce to the case in which the system and solution are synchronized.

Indeed, for (Sy.1), let $\Theta$ be an arbitrary $\theta_{0}$-class. Then there exists a word $s_{\Theta} \in A^{-\mathbb{N}}$ and words $u_{x} \in A^{*}$, for each variable $x$ with $\mathbf{d}_{x} \in \Theta$, such that

$$
\mathbf{d}_{x}=s_{\Theta} u_{x} .
$$

Notice that, if $\mathbf{d}_{x}$ is ultimately periodic, then we may choose $s_{\Theta}$ of the form $u^{-\infty}$ where $u \in A^{+}$is the unique period of $\mathbf{d}_{x}$ which is a Lyndon word. Hence there exists a pseudoword $\rho_{\Theta}$ (whose projection into $\bar{\Omega}_{A} \mathbf{D}$ is $s_{\Theta}$ ) and, for each variable $x$ with $\mathbf{d}_{x} \in \Theta$, there exist pseudowords $\pi_{x}$ such that $\delta(x)=\pi_{x} \rho_{\Theta} u_{x}$. Again proceeding as in the proof of Proposition 6.9 if necessary, we may assume that for each such variable $x$ there is a variable $x^{\prime}$ such that $x x^{\prime}$ occurs in $\mathcal{S}$. We substitute the variables $x$ and $x^{\prime}$ by variables $x_{1}$ and $x_{1}^{\prime}$, respectively, and let $\delta_{1}\left(x_{1}\right)=\pi_{x} \rho_{\Theta}$ and $\delta_{1}\left(x_{1}^{\prime}\right)=u_{x} \delta\left(x^{\prime}\right)$. This defines a solution $\delta_{1}$ of a new system $\delta_{1}$ satisfying condition (Sy.1). Let $M_{1}>M+\left|u_{x}\right|$. If $\delta_{1}^{\prime}=\delta_{1}^{\prime}\left(\delta_{1}, \delta_{1}, M_{1}\right)$ is a $\kappa$-solution of this new system and verifies conditions (TT.1). (TT.5), then by (TT.5) $\delta_{1}^{\prime}\left(x_{1}^{\prime}\right)$ is of form $\delta_{1}^{\prime}\left(x_{1}^{\prime}\right)=u_{x} \pi_{x^{\prime}}^{\prime}$ for some pseudoword $\pi_{x^{\prime}}^{\prime}$, whence we may obtain a $\kappa$-solution $\delta^{\prime}=\delta^{\prime}(\mathcal{S}, \delta, M)$ of $\mathcal{S}$ satisfying conditions (TT.1). (TT.5) by setting $\delta^{\prime}(x)=\delta_{1}^{\prime}\left(x_{1}\right) u_{x}$ and $\delta^{\prime}\left(x^{\prime}\right)=\pi_{x^{\prime}}^{\prime}$. Therefore we may assume that $\mathcal{S}$ verifies condition (Sy.1), Notice that the dual of condition (Sy.1) for confinal words of $K_{\mathcal{S}}$ cannot be assumed since it cannot be guaranteed simultaneously with (Sy.1).

Condition (Sy.2) is a consequence of (Sy.1) in case $\mathbf{w}_{(i, j)}, \mathbf{w}_{(\ell, m)} \in W_{\mathcal{S}}$ are words such that $\mathbf{w}_{(i, j)} \sim \mathbf{w}_{(\ell, m)}$ and $\mathbf{w}_{(i, j)}$ is not left-ultimately periodic (and so nor is $\mathbf{w}_{(\ell, m)}$ ). If $\mathbf{w}_{(i, j)}=\mathbf{d}_{i, j-1} \cdot \mathbf{k}_{i, j}$ is left-ultimately periodic, then $\mathbf{w}_{(\ell, m)}=\mathbf{d}_{\ell, m-1} \cdot \mathbf{k}_{\ell, m}$ is also leftultimately periodic and, by (Sy.1), $\mathbf{d}_{i, j-1}=\mathbf{d}_{\ell, m-1}=u^{-\infty}$ where $u$ is a Lyndon word. Since $\mathbf{w}_{(i, j)} \sim \mathbf{w}_{(\ell, m)}$, then we may assume, without loss of generality, that $\mathbf{k}_{\ell, m}=u^{p} \mathbf{k}_{i, j}$ for some integer $p \geq 0$. So, as in the case of condition (Sy.1), we may transfer the suffix $u^{p}$ from $\delta\left(x_{i, j-1}\right)$ to $\delta\left(x_{i, j}\right)$. This does not change the value of $\mathbf{d}_{i, j-1}$ and makes $\mathbf{k}_{\ell, m}=\mathbf{k}_{i, j}$, so that $\mathbf{w}_{(i, j)}=\mathbf{w}_{(\ell, m)}$ in the new system. We may therefore assume that $($ Sy.2) is verified by $\mathcal{S}$.

Now, notice that by Lemma 4.3 we may assume that a word $\mathbf{w}_{(i, j)}$ belongs to $W_{i}(0)$ if and only if there is a word $\mathbf{w}_{(i, m)}$ in $W_{i}(1)$ such that $\mathbf{w}_{(i, j)} \sim \mathbf{w}_{(i, m)}$. So, the equality $W_{i}(0)=W_{i}(1)$ is a consequence of condition (Sy.2), which shows that condition (Sy.3) may also be assumed.

Let $x=x_{i, j}$ be a variable such that $\delta(x)$ is a $\kappa$-term when projected into $\bar{\Omega}_{A} \mathbf{L S l}$. The set $B_{\delta(x)}$ of non-periodic bi-infinite factors of $\delta(x)$ is finite. Therefore, since each element of $B_{\delta(x)}$ is a factor of $\delta\left(x_{i, 1} \cdots x_{i, p_{i}}\right)$, we may assume, again by Lemma 4.3, that $B_{\delta(x)} \subseteq W_{i}(0)$. Therefore, we may also assume condition (Sy.4).

Finally, suppose that $W_{\mathcal{S}}$ contains a periodic word $\mathbf{w}_{(i, j)}=u^{\infty}$. Then $\mathbf{d}_{i, j-1}=u^{-\infty}$ and $\mathbf{k}_{i, j}=u^{+\infty}$. We let $b_{u}$ be a new letter and we modify the system of equations by introducing a new variable $y_{\ell, m}$, labeled by $b_{u}^{\omega}$, between all pairs of variables $x_{\ell, m-1}$ and $x_{\ell, m}$ (and so, in particular, between $x_{i, j-1}$ and $x_{i, j}$ ) such that $\mathbf{w}_{(\ell, m)}=u^{\infty}$. Iterating this procedure, and since by (Sy.3) $W_{i}(0)=W_{i}(1)$ for every $i$, we obtain a new system $\delta_{1}$ and a new solution $\delta_{1}$ over LSl satisfying condition (Sy.5), If $\delta_{1}^{\prime}=\delta_{1}^{\prime}\left(\mathcal{S}_{1}, \delta_{1}, M\right)$ is a $\kappa$-solution of this new system and verifies conditions (TT.1). (TT.5), then by (TT.2) 
and $\left(\right.$ TT.3) $\mathbf{D} \models \delta_{1}^{\prime}\left(x_{\ell, m-1}\right)=\delta_{1}\left(x_{\ell, m-1}\right)=u^{\omega}$ and $\mathbf{K} \models \delta_{1}^{\prime}\left(x_{\ell, m}\right)=\delta_{1}\left(x_{\ell, m}\right)=u^{\omega}$, and by (TT.4) LSl $=\delta_{1}^{\prime}\left(y_{\ell, m}\right)=\delta_{1}\left(y_{\ell, m}\right)=b_{u}^{\omega}$. Therefore, the restriction of $\delta_{1}^{\prime}$ to $X$ clearly defines a $\kappa$-solution over LSl of the original system $\mathcal{S}$. This proves that condition (Sy.5) may also be assumed and concludes the proof of the proposition.

The objective of the rest of the paper is to prove the TT $\kappa$-reducibility of LSl. We assume therefore that $\mathcal{S}$ is a fixed finite system of word equations of the form

$$
x_{i, 1} \cdots x_{i, p_{i}}=x_{i, p_{i}+1} \cdots x_{i, q_{i}} \quad(i=1, \ldots, h)
$$

where each $x_{i, j}$ is a variable, and let $\delta$ be a solution of $\mathcal{S}$ over $\mathbf{L S I}$ with respect to a pair $(\gamma, \psi)$, labeling each variable by an infinite pseudoword. We further assume that this system and solution are synchronized and that each variable occurs exactly once in $\mathcal{S}$. We also suppose that a positive integer $M$ is fixed (cf. Definition 6.12). The objective is therefore to construct a $\kappa$-solution $\delta^{\prime}=\delta^{\prime}(\mathcal{S}, \delta, M)$ of $\mathcal{S}$ over LSl with respect to $(\gamma, \psi)$ satisfying conditions (TT.1) to (TT.5)

\subsection{Reduction rule}

The algorithm for the construction of the $\kappa$-solution $\delta^{\prime}$ will closely follow some parts of the one used by Teixeira and the first author in [15] to prove the $\kappa$-reducibility of LSl.

We begin by giving a general description of the algorithm. Let $n$ be a sufficiently large integer (to be specified below). For each variable $x \in X$, fix a word $w_{x} \in A^{+}$, given by Lemma 4.4, such that $w_{x} \equiv_{n} \delta(x)$ and $\psi\left(w_{x}\right)=\psi(\delta(x))$. When we are interested in identifying the (unique) place where $x$ occurs in $\mathcal{S}$, say when $x=x_{i, j}$, we will also denote $w_{x_{i, j}}$ by $w_{i, j}$. Each of these words $w_{x}$ will be transformed, according to a process described in Subsection 6.5, into a rank $1 \kappa$-term $\widehat{w_{x}}$ which will be selected to be $\delta^{\prime}(x)$. This process of transformation consists of a single reduction rule, which substitutes certain factors $u$ of $w_{x}$ by certain rank $1 \kappa$-terms $\bar{u}$. So, $\widehat{w_{x}}$ will be a rank $1 \kappa$-term of the form $\widehat{w_{x}}=u_{0}^{\prime} \overline{u_{1}} u_{1}^{\prime} \cdots \overline{u_{m}} u_{m}^{\prime}$ with $w_{x}=u_{0}^{\prime} u_{1} u_{1}^{\prime} \cdots u_{m} u_{m}^{\prime}$. We proceed to describe the reduction rule.

By Lemma 2.2, for each word $u=a_{1} \cdots a_{k} \in A^{+}$of length $k=|S|$, there is a rank 1 $\kappa$-term

$$
\bar{u}=a_{1} \cdots a_{i-1}\left(a_{i} \cdots a_{j}\right)^{\omega} a_{j+1} \cdots a_{k}
$$

such that $\psi(u)=\psi(\bar{u})$. Now, suppose that $u \in A^{+} \backslash A$ is a word such that the semigroup $S$ satisfies $u=u^{\omega}$. Let $v \in A^{+}$be the primitive root of $u$ (i.e., $v$ is the unique primitive word such that $u$ is a power of $v$ ). In this case, we let

$$
\bar{u}=v^{\omega}
$$

so that equality $\psi(u)=\psi(\bar{u})$ also holds in this case. Let $\bar{A}$ be the alphabet

$$
\bar{A}=A \uplus\left\{\bar{u} \mid u \in A^{+} \text {and } \bar{u} \text { is defined }\right\} .
$$

Let $w \in \bar{A}^{+} \backslash A^{+}$. We say that a factor $w^{\prime}$ of $w$ is essential if $w^{\prime}$ is of the form $\overline{u_{1}} z \overline{u_{2}}$, with $\overline{u_{1}}, \overline{u_{2}} \in \bar{A} \backslash A$ and $z \in A^{*}$, and $w^{\prime}$ is not of the form $\bar{u} u^{m} \bar{u}$, with $\bar{u}=v^{\omega}$ given by (6.13) (i.e., $w^{\prime}$ is not equal to $\bar{u}$ over $\mathbf{L S l}$ ). The unique prefix of $w$ of the form $z \bar{u}$, where $\bar{u} \in \bar{A} \backslash A$ and $z \in A^{*}$, is called the essential prefix of $w$. The definition of the essential suffix of $w$ is dual. 
Definition 6.14 (reduction rule) The reduction rule (to be applied on terms in the alphabet $\bar{A}$ ) is the following:

(R) $t_{1} u t_{2} \rightarrow t_{1} \bar{u} t_{2}$, where $t_{1}, t_{2} \in \bar{A}^{*}, u \in A^{+}$and $\bar{u}$ is defined.

Notice that this rule defines a Nœetherian system since it reduces the length of terms in the alphabet $\bar{A}$. Moreover, $\psi\left(t_{1} u t_{2}\right)=\psi\left(t_{1} \bar{u} t_{2}\right)$. Since, by Lemma 4.4, $S$ satisfies $\delta(x)=w_{x}$, this equality will insure that $\psi \circ \delta^{\prime}=\gamma$. Therefore, to make $\delta^{\prime}$ be a $\kappa$ solution of $\mathcal{S}$ over $\mathbf{L S l}$, it suffices to guarantee that the pseudoidentity $\delta^{\prime}\left(x_{i, 1} \cdots x_{i, p_{i}}\right)=$ $\delta^{\prime}\left(x_{i, p_{i}+1} \cdots x_{i, q_{i}}\right)$, that is the pseudoidentity

$$
\widehat{w_{i, 1}} \cdots \widehat{w_{i, p_{i}}}=\widehat{w_{i, p_{i}+1}} \cdots \widehat{w_{i, q_{i}}} \text {, }
$$

holds over LSl for every $i \in\{1, \ldots, h\}$. Notice that each $\widehat{w_{i, j}}$ may be seen as a word over $\bar{A}$. To make (6.14) hold over LI it suffices to assure that the essential prefixes of $\widehat{w_{i, 1}}$ and $\widehat{w_{i, p_{i}+1}}$ are the same, and that the essential suffixes of $\widehat{w_{i, p_{i}}}$ and $\widehat{w_{i, q_{i}}}$ also coincide. Therefore, to guarantee that LSl verifies (6.14) it suffices, by Proposition 4.8, to insure that the words $\widehat{w_{i, 1}} \cdots \widehat{w_{i, p_{i}}}$ and $\widehat{w_{i, p_{i}+1}} \cdots \widehat{w_{i, q_{i}}}$ have the same essential factors.

\subsection{Centers of $\mathcal{S}$}

As mentioned in the previous subsection, the $\kappa$-solution $\delta^{\prime}$ will be defined, for each variable $x$, as $\delta^{\prime}(x)=\widehat{w_{x}}$. Moreover, by definition of the reduction rule, $\widehat{w_{x}}$ will be a rank $1 \kappa$-term of the form $\widehat{w_{x}}=u_{0}^{\prime} \overline{u_{1}} u_{1}^{\prime} \cdots \overline{u_{m}} u_{m}^{\prime}$ where $w_{x}=u_{0}^{\prime} u_{1} u_{1}^{\prime} \cdots u_{m} u_{m}^{\prime}$.

The main objective of this subsection is to determine the prefix $l_{x}=u_{0}^{\prime} u_{1}$ and the suffix $r_{x}=u_{m} u_{m}^{\prime}$ of the word $w_{x}$, that is, is to identify the first and the last occurrences of factors of $w_{x}$ on which rule $(R)$ is going to be applied. These uses of the rule will determine the essential prefix and the essential suffix of $\widehat{w_{x}}$. Notice that $w_{x}$ and $\delta(x)$ have the same prefix and the same suffix of length $n$. So, we will choose a sufficiently large $n$ so that the words $l_{x}$ and $r_{x}$ will be also a prefix and a suffix of $\delta(x)$ (which is equivalent to saying that they are a prefix and a suffix of the projections $\mathbf{k}_{x} \in A^{\mathbb{N}}$ and $\mathbf{d}_{x} \in A^{-\mathbb{N}}$ of $\delta(x)$ into $\bar{\Omega}_{A} \mathbf{K}$ and $\bar{\Omega}_{A} \mathbf{D}$, respectively). Moreover, since by condition (TT.5) we want to preserve prefixes of length $M$, we will impose that $\left|u_{0}^{\prime}\right| \geq M$.

Notice that, if the above $x$ is such that $x=x_{i, j}$ with $j \in\left\{2, \ldots, p_{i}, p_{i}+2, \ldots, q_{i}\right\}$ and $\widehat{w_{i, j-1}}=v_{0}^{\prime} \overline{v_{1}} v_{1}^{\prime} \cdots \overline{v_{\ell}} v_{\ell}^{\prime}$, then $\overline{v_{\ell}} v_{\ell}^{\prime} u_{0}^{\prime} \overline{u_{1}}$ (which is the product of the essential suffix of $\widehat{w_{i, j-1}}$ and the essential prefix of $\widehat{w_{i, j}}$ ) is an essential factor of $\widehat{w_{i, j-1}} \widehat{w_{i, j}}$ and so, as mentioned at the end of the previous subsection, we want to make $\overline{v_{\ell}} v_{\ell}^{\prime} u_{0}^{\prime} \overline{u_{1}}$ occur, in the equality (6.14), in the opposite side of the variable $x$. The finite word $v_{\ell} v_{\ell}^{\prime} u_{0}^{\prime} u_{1}$, that is $r_{i, j-1} l_{i, j}$, will be called a center of the system.

Let $\Theta$ be a $\theta_{1}$-class. Then there exists a word $p_{\Theta} \in A^{\mathbb{N}}$ and words $p_{x} \in A^{*}$, for each variable $x$ with $\mathbf{k}_{x} \in \Theta$, such that

$$
\mathbf{k}_{x}=p_{x} p_{\Theta}
$$

We fix a factorization as in (6.15) satisfying certain conditions. First, we assume that the length of each word $p_{x}$ is at least $M$. Next, if $y$ is a variable such that $\mathbf{k}_{x}=\mathbf{k}_{y}$, then we let $p_{x}=p_{y}$. Furthermore, if $\mathbf{k}_{x}$ is ultimately periodic, then we assume that $p_{\Theta}=u^{+\infty}$ where $u \in A^{+}$is the unique period of $\mathbf{k}_{x}$ which is a Lyndon word. Other conditions that (6.15) must satisfy will be imposed below. 
On the other hand, and since condition (Sy.1) is valid, for each $\theta_{0}$-class $\Theta$ and each variable $x$ such that $\mathbf{d}_{x} \in \Theta$, there is a factorization

$$
\mathbf{d}_{x}=s_{\Theta},
$$

with $s_{\Theta} \in A^{-\mathbb{N}}$ such that, if $\mathbf{d}_{x}$ is ultimately periodic, then $s_{\Theta}=v^{-\infty}$ where $v \in A^{+}$is the unique period of $\mathbf{d}_{x}$ which is a Lyndon word.

Definition 6.15 (words $l_{x}, \widehat{l_{x}}, r_{x}, \widehat{r_{x}}, c_{(i, j)}, \widehat{c_{(i, j)}}$ and set $C_{\mathcal{S}}$ ) Let $x$ be a variable. We fix a prefix $l_{x}$ of $\mathbf{k}_{x}$, and a rank $1 \kappa$-term $\widehat{l_{x}}$, as follows

$$
l_{x}=p_{x} u_{\Theta} \quad \text { and } \quad \widehat{l_{x}}=p_{x} \overline{u_{\Theta}}
$$

where:

- $\Theta$ is the $\theta_{1}$-class of $x$;

- if $p_{\Theta}$ is non-ultimately periodic, then $u_{\Theta}$ is the prefix of $p_{\Theta}$ of length $k$ and $\overline{u_{\Theta}}$ is given by $(6.12)$;

- if $p_{\Theta}=u^{+\infty}$ is ultimately periodic, then $u_{\Theta}=u^{\ell}$ for some (sufficiently large) integer $\ell>0$ such that $S$ satisfies $u^{\omega}=u^{\ell}$ and $\overline{u_{\Theta}}=u^{\omega}$ is given by (6.13). In this case, we say that $l_{x}$ is ultimately periodic and the word $u$ is called the period of $l_{x}$.

Notice that, in particular, the words $l_{x}$ may be chosen so that

$$
\mathbf{k}_{x}=\mathbf{k}_{y} \Longleftrightarrow l_{x}=l_{y} \text { and } \widehat{l_{x}}=\widehat{l_{y}} .
$$

We also fix a suffix $r_{x}$ of $\mathbf{d}_{x}$, and a rank $1 \kappa$-term $\widehat{r_{x}}$, as follows

$$
r_{x}=v_{\Theta} \quad \text { and } \quad \widehat{r_{x}}=\overline{v_{\Theta}^{\prime}} v_{\Theta}^{\prime \prime}
$$

where:

- $\Theta$ is the $\theta_{0}$-class of $x$;

- if $s_{\Theta}$ is non-ultimately periodic, then $v_{\Theta}=v_{\Theta}^{\prime} v_{\Theta}^{\prime \prime}$ is a suffix of $s_{\Theta}$, where $\left|v_{\Theta}^{\prime}\right|=k$, and $\overline{v_{\Theta}^{\prime}}$ is determined by (6.12);

- if $s_{\Theta}=v^{-\infty}$ is ultimately periodic, then $v_{\Theta}=v^{m}$ for some integer $m>0$ such that $S$ satisfies $v^{\omega}=v^{m}$ and $\overline{v_{\Theta}}=v^{\omega}$ is determined by (6.13). In this case, we say that $r_{x}$ is (ultimately) periodic and the word $v$ is called the period of $r_{x}$.

Notice that, in particular, the words $r_{x}$ may be chosen so that

$$
\mathbf{d}_{x}=\mathbf{d}_{y} \Longleftrightarrow r_{x}=r_{y} \text { and } \widehat{r_{x}}=\widehat{r_{y}} \text {. }
$$

Finally, for a pair $(i, j)$ of integers with $i \in\{1, \ldots, h\}$ and $j \in\left\{2, \ldots, p_{i}, p_{i}+2, q_{i}\right\}$, we let

$$
c_{(i, j)}=r_{i, j-1} l_{i, j} \quad \text { and } \quad \widehat{c_{(i, j)}}=\widehat{r_{i, j-1}} \widehat{l_{i, j}}
$$

and denote by $C_{\mathcal{S}}$ the set of all such words $c_{(i, j)}$. 
Notice that the word $c_{(i, j)}$ is a center of $\mathbf{w}_{(i, j)}$. Moreover,

$$
\mathbf{w}_{(i, j)}=\mathbf{w}_{(\ell, m)} \Longleftrightarrow c_{(i, j)}=c_{(\ell, m)} \Longleftrightarrow \widehat{c_{(i, j)}}=\widehat{c_{(\ell, m)}} .
$$

The set $C_{\mathcal{S}}$ will be said to be the set of centers of $\mathcal{S}$. A center $c_{(i, j)}$ will be called ultimately periodic when the word $\mathbf{w}_{(i, j)}$ is ultimately periodic (or equivalently $r_{i, j-1}$ and $l_{i, j}$ are ultimately periodic), in which case $c_{(i, j)}$ is of the form $v^{m} p_{i, j} u^{\ell}$. Notice that, by (Sy.5), none of the words $\mathbf{w}_{(i, j)}$ is a periodic word. For this reason, we will say that the ultimately periodic centers $c_{(i, j)}=v^{m} p_{i, j} u^{\ell}$ of $C_{\mathcal{S}}$ are non-periodic, meaning that either $v \neq u$ or, $v=u$ and $p_{i, j}$ is not a power of $v$.

The words $c_{(i, j)}$ and $r_{x}$ must satisfy also the three following conditions:

(Ce.1) If $c_{(\ell, m)}$ and $\mathbf{w}_{(i, j)}$ are ultimately periodic and $\mathbf{w}_{(\ell, m)} \neq \mathbf{w}_{(i, j)}$, then $c_{(\ell, m)}$ is not a factor of $\mathbf{w}_{(i, j)}$;

(Ce.2) For every $\mathbf{w}_{(i, j)} \in W_{\mathcal{S}}$ and every variable $x$, if $\mathbf{w}_{(i, j)}$ is not a bi-infinite factor of $\delta(x)$, then $c_{(i, j)}$ is not a factor of $\delta(x)$;

(Ce.3) $\left|r_{x}\right| \geq Q$ for every variable $x$, where $Q$ is a positive integer in the conditions of Lemma 5.3 for $B=W_{\mathcal{S}}$.

Since the word $c_{(i, j)}$ is a center of the element $\mathbf{w}_{(i, j)}$ of $B=W_{\mathcal{S}}$, and since $\left|r_{i, j}\right| \geq Q$ by the last condition above, Lemma 5.3 guarantees the existence of a right-extension $\overrightarrow{c_{(i, j)}}$ of $c_{(i, j)}$ such that, for every $\mathbf{w}_{(i, j)}, \mathbf{w}_{(\ell, m)} \in W_{\mathcal{S}}$, and for every word $w \in A^{+}$,

if two distinct occurrences of $c_{(i, j)}$ and $c_{(\ell, m)}$ are allowed in $w$ relative to $\overrightarrow{c_{(i, j)}}$ and $\overrightarrow{c_{(\ell, m)}}$ respectively, then these occurrences of $c_{(i, j)}$ and $c_{(\ell, m)}$ are disjoint.

The right-extension $\overrightarrow{c_{(i, j)}}$ of $c_{(i, j)}$ will be called the extended-center of $\mathbf{w}_{(i, j)}$. Finally, we fix the integer $n$, mentioned in the beginning of Subsection 6.3 .

Definition 6.16 (constants $L$ and $n$ and words $w_{x}$ ) Let $L$ be an integer greater than the lengths of all words $l_{x}, r_{x}, c_{(i, j)}$ and $\overrightarrow{c_{(i, j)}}$. Then we fix an integer $n>3 L+k^{\prime \prime}$ and, for each variable $x$, we fix a word $w_{x} \in A^{+}$such that $w_{x} \equiv_{n} \delta(x)$ and $\psi\left(w_{x}\right)=\psi(\delta(x))$.

\subsection{Transformations on the words $w_{x}$}

In this subsection we describe the algorithm that permits to transform each word $w_{x}$ into a rank $1 \kappa$-term $\widehat{w_{x}}$, which is then defined to be $\delta^{\prime}(x)$. Recall that $l_{x}$ is a prefix and $r_{x}$ is a suffix of $w_{x}$, so that we can write

$$
w_{x}=l_{x} w_{x}^{\prime} r_{x}=p_{x} u_{\Theta} w_{x}^{\prime} v_{\Theta}
$$

for some $w_{x}^{\prime} \in A^{+}$.

A variable $y$ such that $\delta(y)$ is a $\kappa$-term when projected into $\bar{\Omega}_{A} \mathbf{L S l}$ will be called from now on a $\kappa$-variable. Although our procedure to obtain $\widehat{w_{x}}$ is generic, we prefer to present first the suggestive case in which $x$ is a $\kappa$-variable. We begin by illustrating this case with an example. 
Example 6.17 Suppose that $x$ is a variable such that

$$
\mathbf{L S} \mathbf{l} \models \delta(x)=d^{\omega} a d^{\omega} b d^{\omega}
$$

with $a, b, d \in A$, so that $B_{\delta(x)}=\left\{d^{-\infty} a d^{+\infty}, d^{-\infty} b d^{+\infty}\right\} \subseteq W_{\mathcal{S}}$ by (Sy.4). Hence, by Definition 6.15, $d^{\ell} d^{m} a d^{\ell}, d^{\ell} d^{m} b d^{\ell} \in C_{\mathcal{S}}$ for some integers $m, \ell$ such that $S$ satisfies $d^{\omega}=d^{\ell}$.

Notice that (6.25) does not mean that $\delta(x)$ is a $\kappa$-term. This is illustrated by the following example (which emerged in a collaboration of the first author with J. Almeida and $M$. Zeitoun): $\delta(x)$ is an accumulation point in $\bar{\Omega}_{A} \mathbf{S}$ of the sequence $\left(\pi_{i}\right)_{i}$ defined by $\pi_{i}=d^{\omega} a_{1} d^{\omega} a_{2} d^{\omega} \cdots a_{i} d^{\omega}$, where $a_{1} a_{2} a_{3} \cdots=$ abaababaabaababaababa $\cdots \in\{a, b\}^{\mathbb{N}}$ is the Fibonacci word.

By definition, $w_{x}$ verifies $\delta(x) \equiv_{n} w_{x}$. Hence, there exist positive integers $p, i_{1}, \ldots, i_{p}$ such that $w_{x}=d^{i_{1}} b_{1} d^{i_{2}} \cdots b_{p} d^{i_{p}}$ with $\left\{b_{1}, \ldots, b_{p}\right\}=\{a, b\}$. By the choice of $n$ it follows that $l_{x}=r_{x}=d^{\ell}$ and $w_{x}=l_{x} d^{i_{1}^{\prime}} c_{1} d^{i_{2}^{\prime}} \cdots c_{p} d^{i^{\prime}} r_{x}$ with $c_{i}=d^{\ell} d^{m} b_{i} d^{\ell}$. We then let

$$
\widehat{w_{x}}=\widehat{l_{x}} d^{i_{1}^{\prime}} \widehat{c_{1}} d^{i_{2}^{\prime}} \ldots \widehat{c_{p}} d^{i_{p}^{\prime}} \widehat{r_{x}}=d^{\omega} d^{i_{1}^{\prime}} d^{\omega} d^{m} b_{1} d^{\omega} d^{i_{2}^{\prime}} \cdots d^{\omega} d^{m} b_{p} d^{\omega} d^{i_{p}^{\prime}} d^{\omega}
$$

which is clearly equal to $\delta(x)$ over LS1. Thus $\delta^{\prime}(x)=\widehat{w_{x}}$ verifies (TT.4).

First Case: $x$ is a $\kappa$-variable. Notice that this is equivalent to say that $\mathbf{L S l} \models \delta(x)=t$ for some $\kappa$-term $t=u_{0}^{\prime} u_{1}^{\omega} u_{1}^{\prime} u_{2}^{\omega} \cdots u_{p}^{\omega} u_{p}^{\prime}$ in reduced form. Hence, each $u_{i}$ is a Lyndon word and $B_{\delta(x)}=\left\{\mathbf{w}_{i} \mid i=1, \ldots, p-1\right\}$, where $\mathbf{w}_{i}=u_{i}^{-\infty} u_{i}^{\prime} u_{i+1}^{+\infty}$. Therefore, by Definition 6.15 and since system $\mathcal{S}$ is synchronized, we may assume that $u_{p}^{\prime}$ is the empty word and that, for each $i \in\{1, \ldots, p-1\}$, there is some center $c_{i} \in C_{\mathcal{S}}$ of the form $c_{i}=u_{i}^{\ell_{i}} u_{i}^{m_{i}} u_{i}^{\prime} u_{i+1}^{\ell_{i+1}}$ for some integers $m_{i}, \ell_{i}$ such that $S$ satisfies $u_{i}^{\omega}=u_{i}^{\ell_{i}}$. Moreover, by $(\overline{6.22}), c_{i}=c_{j}$ if and only if $\mathbf{w}_{i}=\mathbf{w}_{j}$.

Lemma 6.18 Every occurrence of a center $c_{i}$ in $w_{x}$ is an allowed occurrence disjoint from $l_{x}$ and $r_{x}$.

Proof. Fix an occurrence $c_{i}=w_{x}\left[m, m^{\prime}\right]$ of a center $c_{i}=u_{i}^{\ell_{i}} u_{i}^{m_{i}} u_{i}^{\prime} u_{i+1}^{\ell_{i+1}}$ in $w_{x}$. The fact that $\delta(x) \equiv_{n} w_{x}$ and $\mathbf{L S l} \models \delta(x)=t$ implies that $w_{x}$ and $u_{0}^{\prime} u_{1}^{+\infty}$ have the same prefix of length $n$. Now, we may assume that $\ell_{i}$ is greater than $\left|u_{0}^{\prime}\right|$ because it may be chosen as large as we want. On the other hand $c_{i}$ is non-periodic, by (Sy.5), and $n>3 \max \left\{\left|l_{x}\right|,\left|c_{i}\right|\right\}$. Therefore, the occurrence of $c_{i}$ is necessarily disjoint from $l_{x}$. Symmetrically, every occurrence of $c_{i}$ is disjoint from $r_{x}$. Moreover since $n>3\left|\overrightarrow{c_{i}}\right|$, for the extended center $\overrightarrow{c_{i}}$ of $c_{i}$, and $\left|\overrightarrow{c_{i}}\right|>\left|c_{i}\right|, c_{i}$ does not occur in the suffix of $w_{x}$ of length $2\left|\overrightarrow{c_{i}}\right|$, whence the fixed occurrence of $c_{i}$ admits a right-extension $c_{i}^{\prime}=w_{x}\left[m, m^{\prime \prime}\right]$ such that $\left|c_{i}^{\prime}\right|=\left|\overrightarrow{c_{i}}\right|$. Notice that $c_{i}^{\prime}=c_{i} v=u_{i}^{\ell_{i}} u_{i}^{m_{i}} u_{i}^{\prime} u_{i+1}^{\ell_{i+1}} v$ for some word $v \in A^{+}$, and that $c_{i}^{\prime}$ is a factor of $t$ since $\delta(x) \equiv_{n} w_{x}$. Therefore $c_{i}^{\prime}$ is a factor of some bi-infinite factor $\mathbf{w}_{j}=u_{j}^{-\infty} u_{j}^{\prime} u_{j+1}^{+\infty}$ of $t$. Since $\mathbf{w}_{i}$ is non-periodic, there is an integer $s>0$ such that $u_{i}^{s} u_{i}^{\prime} u_{i+1}^{s}$ is not a factor of $u_{j}^{-\infty}$ nor of $u_{j+1}^{+\infty}$. Therefore, since $\ell_{i}$ and $\ell_{i+1}$ are arbitrarily large, we may conclude that the prefix $u^{\prime}$ of $c_{i}$ (and of $u_{i}^{\ell_{i}}$ ) of length $\left|u_{i}\right|+\left|u_{j}\right|$ occurs in $u_{j}^{-\infty}$. Thus, $u^{\prime}$ is a common prefix of a power of $u_{i}$ and of a power of a conjugate $\bar{u}_{j}$ of $u_{j}$. Hence, by Fine and Wilf's Theorem, $u_{i}$ and $\bar{u}_{j}$ are powers of the same word. Since $u_{i}$ and $\bar{u}_{j}$ are both primitive words it follows that $u_{i}=\bar{u}_{j}$ and, consequently, $\bar{u}_{j}$ is a Lyndon word. But $\bar{u}_{j}$ is a conjugate of the Lyndon word $u_{j}$, whence $\bar{u}_{j}=u_{j}$. We conclude therefore that $u_{i}=u_{j}$. By symmetry, we also deduce that a sufficiently large suffix of $c_{i}$ occurs in $u_{j+1}^{+\infty}$, which as above permits to conclude that $u_{i+1}=u_{j+1}$. Moreover this 
permits also to deduce, together with the fact that $c_{i}^{\prime}=c_{i} v$ is a right-extension of $c_{i}$, that the suffix $v$ of $c_{i}^{\prime}$ is of the form $v=u_{i+1}^{\ell_{i+1}^{\prime}} \bar{u}$ for some integer $\ell_{i+1}^{\prime}>0$ and some prefix $\bar{u}$ of $u_{i+1}$. Therefore $c_{i}^{\prime}=\overrightarrow{c_{i}}$, which proves that the fixed occurrence $c_{i}=w_{x}\left[m, m^{\prime}\right]$ is an allowed occurrence and concludes the proof.

A consequence of this lemma is that the occurrences of centers $c_{i}$ in $w_{x}$ are pairwise disjoint. Moreover, with similar arguments as in the proof of the lemma, one can show that the word $w_{x}$ can be written in the two following forms

$$
w_{x}=v_{0}^{\prime} v_{1}^{i_{1}} v_{1}^{\prime} v_{2}^{i_{2}} \cdots v_{q}^{i_{q}}=l_{x} v_{1}^{i_{1}^{\prime}} c_{f_{1}} v_{2}^{i_{2}^{\prime}} \cdots c_{f_{q}} v_{q}^{i_{q}^{\prime}} r_{x}
$$

where:

- $q \geq 1, v_{0}^{\prime}=u_{0}^{\prime}, v_{1}=u_{1}, v_{q}=u_{p}$;

- $\left\{v_{i}^{-\infty} v_{i}^{\prime} v_{i+1}^{+\infty} \mid i=1, \ldots, q-1\right\}=B_{\delta(x)}$;

- $l_{x}=p_{x} u_{\Theta}$ with $p_{x}=v_{0}^{\prime} v_{1}^{\ell_{1}^{\prime}}$ for some integer $\ell_{1}^{\prime} \geq 0$ and $u_{\Theta}=v_{1}^{\ell_{1}}, r_{x}=v_{\Theta}=v_{q}^{\ell_{q}}$;

- $c_{f_{i}}=v_{i}^{\ell_{i}} v_{i}^{j_{i}} v_{i}^{\prime} v_{i+1}^{\ell_{i+1}}$.

We let

$$
\begin{aligned}
\widehat{w_{x}} & =\widehat{l_{x}} v_{1}^{i_{1}^{\prime}} \widehat{{c_{1}}_{1}} v_{2}^{i_{2}^{\prime}} \cdots \widehat{c_{f_{q}}} v_{q}^{i_{q}^{\prime}} \widehat{r_{x}} \\
& =p_{x} \overline{u_{\Theta}} v_{1}^{i_{1}^{\prime}} \overline{v_{1}^{\ell_{1}}} v_{1}^{j_{1}} v_{1}^{\prime} \overline{v_{2}^{\ell_{2}}} v_{2}^{i_{2}^{\prime}} \cdots \overline{v_{q-1}^{\ell_{q}}} v_{q-1}^{j_{q-1}} v_{q-1}^{\prime} \overline{v_{q}^{\ell_{q}}} v_{q}^{i_{q}^{\prime}} \overline{v_{\Theta}} \\
& =v_{0}^{\prime} v_{1}^{\ell_{1}^{\prime}} v_{1}^{\omega} v_{1}^{i_{1}^{\prime}} v_{1}^{\omega} v_{1}^{j_{1}} v_{1}^{\prime} v_{2}^{\omega} v_{2}^{i_{2}^{\prime}} \cdots v_{q-1}^{\omega} v_{q-1}^{j_{q-1}} v_{q-1}^{\prime} v_{q}^{\omega} v_{q}^{i_{q}^{\prime}} v_{q}^{\omega},
\end{aligned}
$$

from which it follows immediately that LSl $\models \widehat{w_{x}}=v_{0}^{\prime} v_{1}^{\omega} v_{1}^{\prime} v_{2}^{\omega} \cdots v_{q-1}^{\omega} v_{q-1}^{\prime} v_{q}^{\omega}$. Now, Proposition 4.8 together with the equalities above, show that $\widehat{w_{x}}=\delta(x)$ over LSl. Therefore, letting $\delta^{\prime}(x)=\widehat{w_{x}}$, conditions (TT.1) to (TT.4) hold for this variable $x$.

To verify that (TT.5) also holds for this variable $x$, suppose that $\delta(x)=w_{1} \pi$, where $w_{1} \in A^{*}$ and $\pi \in \bar{\Omega}_{A} \mathbf{S}$ with $\left|w_{1}\right| \leq M$. Since $\left|p_{x}\right| \geq M$ by definition (see the assumptions after $(6.15)$ ), there is a factorization $p_{x}=w_{1} w_{1}^{\prime}$, and we may certainly assume that $w_{x}$ was chosen in such a way that $\psi(\pi)=\psi\left(w_{1}^{\prime} u_{\Theta} v_{1}^{i_{1}^{\prime}} c_{f_{1}} v_{2}^{i_{2}^{\prime}} \cdots c_{f_{q}} v_{q}^{i_{q}^{\prime}} r_{x}\right)$. Hence $\delta^{\prime}(x)=w_{1} \pi^{\prime}$, where $\pi^{\prime}=w_{1}^{\prime} \overline{u_{\Theta}} v_{1}^{i_{1}^{\prime}} \widehat{c_{f_{1}}} v_{2}^{i_{2}^{\prime}} \cdots \widehat{c_{f_{q}}} v_{q}^{i_{q}^{\prime}} \widehat{r_{x}} \in \Omega_{A}^{\kappa} \mathbf{S}$, so that

$$
\psi(\pi)=\psi\left(w_{1}^{\prime} u_{\Theta} v_{1}^{i_{1}^{\prime}} c_{f_{1}} v_{2}^{i_{2}^{\prime}} \cdots c_{f_{q}} v_{q}^{i_{q}^{\prime}} r_{x}\right)=\psi\left(\pi^{\prime}\right) .
$$

Now, suppose that $y$ is another variable and that $\delta(y)=w_{2} \rho$, where $w_{2} \in A^{*}$ and $\rho \in \bar{\Omega}_{A} \mathbf{S}$ are such that $\left|w_{2}\right| \leq M$ and LSl $\models=\pi$. Since $\delta(x)=w_{1} \pi, \delta^{\prime}(x)=w_{1} \pi^{\prime}$ and LSI $\models \delta(x)=\delta^{\prime}(x)$, it follows from Corollary 4.2 that LSl $\models \pi=\pi^{\prime}$, whence $\mathbf{L S l}=\delta(y)=w_{2} \rho=w_{2} \pi=w_{2} \pi^{\prime}$. Therefore, $\delta(y)$ is a $\kappa$-term when projected into $\bar{\Omega}_{A} \mathbf{L S l}$ and, as above for $x$, we deduce that $\mathbf{L S} \models=\delta^{\prime}(y)=\delta(y)$. Hence, $\delta^{\prime}(y)=w_{2} \rho^{\prime}$ for some $\rho^{\prime} \in \Omega_{A}^{\kappa} \mathbf{S}$, and $\mathbf{L S}=\rho^{\prime}=\rho=\pi=\pi^{\prime}$, which proves that (TT.5) is verified by $x$.

Second Case: $x$ is not a $\kappa$-variable. In this case we apply the following algorithm: 
Step 1. We begin by localizing in $w_{x}$ every allowed occurrences of the centers $c_{(\ell, m)}$ of $\mathcal{S}$ (relative to its right-extensions $\overrightarrow{c_{(\ell, m)}}$ ). As observed above, the allowed occurrences of centers are disjoint. Moreover, since we can choose the right-extensions $\overrightarrow{c_{(\ell, m)}}$ as wider as we want, we may assume that any allowed occurrence in $w_{x}$ of a center is disjoint from the suffix $r_{x}$ of $w_{x}$. On the other hand, we claim that any allowed occurrence in $w_{x}$ of a center is disjoint from the prefix $l_{x}$ of $w_{x}$. Indeed, suppose that $x=x_{i, j}$. We may assume that $j \notin\left\{1, p_{i}+1\right\}$ since, otherwise, we could reduce to a new system by multiplying (on the left) the $i$-th equation of $\mathcal{S}$ by a new variable \# labeled by $b^{\omega}$ with $b$ a new letter. Therefore $c_{(i, j)}=r_{i, j-1} l_{x}$. If there would exist an allowed occurrence in $w_{x}$ of a center $c_{(\ell, m)}$, not disjoint from the prefix $l_{x}$, then there would be an allowed occurrence in $r_{i, j-1} w_{x}$ of $c_{(\ell, m)}$, not disjoint from the prefix $c_{(i, j)}=r_{i, j-1} l_{x}$ of $r_{i, j-1} w_{x}$. This is not possible, thus proving the claim. Notice that we may assume not only that the occurrences of allowed centers are disjoint, but that they are, furthermore, separated by factors of length at least $3 k-1$ (for this it would suffice to consider wider extended centers).

This procedure determines a unique factorization

$$
w_{x}=l_{x} w_{x, 0} c_{x, 1} w_{x, 1} c_{x, 2} \cdots c_{x, i_{x}} w_{x, i_{x}} r_{x}
$$

of $w_{x}$ such that

- $i_{x} \geq 0$

- $c_{x, 1}, \ldots, c_{x, i_{x}}$ are centers of $\mathcal{S}$, called the allowed centers of $w_{x}$;

- $w_{x, 0}, w_{x, 1}, \ldots, w_{x, i_{x}} \in A^{*}$ with $\left|w_{x, j}\right| \geq 3 k-1$;

- the factorization contains every allowed occurrences of centers of $\mathcal{S}$ in $w_{x}$.

We then use this factorization of $w_{x}$ to transform $w_{x}$ into the following $\kappa$-term

$$
w_{x}(1)=\widehat{l_{x}} w_{x, 0} \widehat{c_{x, 1}} w_{x, 1} \widehat{c_{x, 2}} \cdots \widehat{c_{x, i_{x}}} w_{x, i_{x}} \widehat{r_{x}} .
$$

This concludes the first step of the algorithm.

Notice that in the First Case (see equation (6.27) above), the algorithm stopped at this point. That was because $\widehat{w_{x}}$ had the same bi-infinite factors (all of them ultimately periodic) and the same projection over $\mathbf{L I}$ as $\delta(x)$, and that was the objective. In this case we need to go further and transform also (some of) the words $w_{x, j}$.

Notice also that the need to localize every allowed occurrences of centers $c_{(\ell, m)}$ in each $w_{x}$, which is illustrated in (6.26) for $\kappa$-variables, is to guarantee that both sides of (6.14) will have the same essential factors, i.e., factors of the form $\bar{u} z \bar{v}$. Indeed, each allowed occurrence of $c_{(\ell, m)}$ will be transformed into $\widehat{c_{(\ell, m)}}$ which is of this type $\bar{u} z \bar{v}$. On the other hand $c_{(\ell, m)}$ has an allowed occurrence in the word $w_{i, 1} \cdots w_{i, p_{i}}$ if and only if it has an allowed occurrence in $w_{i, p_{i}+1} \cdots w_{i, q_{i}}$, which guarantees that $\widehat{c_{(\ell, m)}}$ is a factor of $\widehat{w_{i, 1}} \cdots \widehat{w_{i, p_{i}}}$ if and only if it is a factor of $\widehat{w_{i, p_{i}+1}} \cdots \widehat{w_{i, q_{i}}}$. However, the number of allowed occurrences of $c_{(\ell, m)}$ in $w_{i, 1} \cdots w_{i, p_{i}}$ and $w_{i, p_{i}+1} \cdots w_{i, q_{i}}$ may be very different. For instance, $w_{i, 1} \cdots w_{i, p_{i}}$ may have exactly one occurrence while $w_{i, p_{i}+1} \cdots w_{i, q_{i}}$ may have many ones; therefore, in this case, if we would not replace each allowed occurrence of $c_{(\ell, m)}$ in $w_{i, p_{i}+1} \cdots w_{i, q_{i}}$ by $\widehat{c_{(\ell, m)}}$, we could risk to create, in other steps of the algorithm, new essential factors in $\widehat{w_{i, p_{i}+1}} \cdots \widehat{w_{i, q_{i}}}$ which could not be obtained in $\widehat{w_{i, 1}} \cdots \widehat{w_{i, p_{i}}}$. 
Step 2. In this step we transform the words $w_{x, j}\left(j \in\left\{0, \ldots, i_{x}\right\}\right)$ in factorization (6.29) of $w_{x}(1)$. In fact, not all these words will be transformed. For each $q \in\left\{1, \ldots, i_{x}\right\}, c_{x, q}$ is some center $c_{(\ell, m)}$ and $c_{(\ell, m)}=r_{\ell, m-1} l_{\ell, m}$. We then denote $r_{x, q}=r_{\ell, m-1}$ and $l_{x, q}=l_{\ell, m}$ so that $c_{x, q}=r_{x, q} l_{x, q}$, and denote furthermore $r_{x, i_{x}+1}=r_{x}$ and $l_{x, 0}=l_{x}$. Following the procedure of the First Case, we will not transform the words $w_{x, j}$ for which $l_{x, j}$ and $r_{x, j+1}$ are ultimately periodic with the same period, say $u$, and $w_{x, j}$ is a power of $u$. Notice that in this case

$$
\mathbf{L S l}=\widehat{l_{x, j}} w_{x, j} \widehat{r_{x, j+1}}=\widehat{l_{x, j}} .
$$

So, we consider a word $w_{x, j}\left(j \in\left\{0, \ldots, i_{x}\right\}\right)$ which is not of this form and show how to transform it into a rank $1 \kappa$-term $\widehat{w_{x, j}}$. This will be done in four substeps, which closely follow [15, Subsection 6.5].

Step 2.1. Consider the marked factorization, described in Subsection 5.2 ,

$$
w_{x, j}=w_{0} v_{1} w_{1} v_{2} \cdots v_{q} w_{q}
$$

of $w_{x, j}$. By definition of marked factors, if $q \geq 1$, then $\left|v_{i}\right| \geq 2 k$ for every $i \in\{1, \ldots, q\}$, so that we can write $v_{i}=v_{i, 1} v_{i, 2} v_{i, 3}$ for some words $v_{i, 1}, v_{i, 2}$ and $v_{i, 3}$ with $\left|v_{i, 1}\right|=\left|v_{i, 3}\right|=$ $k$. We define $\widehat{v}_{i}=\overline{v_{i, 1}} v_{i, 2} \overline{v_{i, 3}}$, and let $w_{x, j}(1)$ be the following rank $1 \kappa$-term

$$
w_{x, j}(1)=w_{0} \widehat{v_{1}} w_{1} \widehat{v_{2}} w_{2} \cdots w_{q-1} \widehat{v_{q}} w_{q} .
$$

Step 2.2. It is clear, from the definition of free factors, that each $w_{i}$ is either the empty word (this can only happen for $i=0$ or $i=q$ ), or it has length greater than $k$ (in fact it is much larger than $k$ ). So, if $w_{0}$ is not the empty word, we define

$$
\widehat{w_{0}}=\overline{w_{0,1}} w_{0,2}
$$

where $w_{0,1}$ is the prefix of length $k$ of $w_{0}$ and $w_{0}=w_{0,1} w_{0,2}$. Symmetrically, if $w_{q} \neq 1$, we let

$$
\widehat{w_{q}}=w_{q, 1} \overline{w_{q, 2}}
$$

where $w_{q, 2}$ is the suffix of length $k$ of $w_{q}$ and $w_{q}=w_{q, 1} w_{q, 2}$. Now, let

$$
w_{x, j}(2)=\widehat{w_{0}} \widehat{v_{1}} w_{1} \widehat{v_{2}} w_{2} \cdots w_{q-1} \widehat{v_{q}} \widehat{w_{q}} \text {. }
$$

Step 2.3. Let $y \in A^{+}$be a factor of $w_{x, j}$ such that $2 k \leq|y|<3 k$. Two cases may arise.

(Case I) Every extension of length $3 k-1$ in $w_{x, j}$, of an occurrence of $y$, is a marked occurrence (see Subsection 5.2). In this case every occurrence of $y$ in $w_{x, j}$ is contained in the marked factors.

(Case II) There exists a free occurrence in $w_{x, j}$ of an extension $\tilde{y}$, of length $3 k-1$, of an occurrence of $y$. In this case, by definition of free occurrence (cf. Subsection 5.2), there exists a $k^{\prime \prime}$-neighborhood $v$ of $\tilde{y}$ such that $v$ is $k^{\prime}$ abundant. In particular, $\operatorname{docc}(\tilde{y}, v) \geq k^{\prime}$. Furthermore, every occurrence of a factor $z$ of length $3 k-1$ in the $k^{\prime \prime}$-neighborhood $v$ is free. This means that at least $k^{\prime}-2$ of the disjoint occurrences of $\tilde{y}$ in $v$ occur disjoint from the marked factors. More precisely, there exists an integer $0 \leq i \leq q \operatorname{such}$ that $\operatorname{docc}\left(\tilde{y}, w_{i}\right) \geq k^{\prime}-2$. Hence, since $y$ is a factor of $\tilde{y}$, $\operatorname{docc}\left(y, w_{i}\right) \geq k^{\prime}-2$. In this case, we say that $y$ has a free occurrence in $w_{x, j}$. 
Consider the set $F$ of all factors $y$ of $w_{x, j}$ such that $2 k \leq|y|<3 k$ and $y$ has a free occurrence in $w_{x, j}$. By the second case above, for each $y \in F$ there exists an integer $0 \leq i \leq q \operatorname{such}$ that $\operatorname{docc}\left(y, w_{i}\right) \geq k^{\prime}-2$. Therefore, the choice of $k^{\prime}$ allows us to select an occurrence for each $y \in F$ in such a way that these occurrences are pairwise disjoint. These occurrences are selected in the factors $w_{i}$ and, if $i=0$ or $i=q$, then we can select them, respectively, in $w_{0,2}$ and in $w_{q, 1}$. Since $2 k \leq|y|<3 k$, we can write

$$
y=y_{1} y_{2} y_{3}
$$

for some words $y_{1}, y_{2}$ and $y_{3}$ with $\left|y_{1}\right|=\left|y_{3}\right|=k$. We substitute in $w_{x, j}(2)$ the selected occurrence of $y$ by $\widehat{y}=\overline{y_{1}} y_{2} \overline{y_{3}}$. We then obtain a term $w_{x, j}(3)$.

Step 2.4. In this step, we admit the substitution of any occurrence, in the factors of $w_{x, j}(3)$ which were obtained from the transformations on the words $w_{i}$, of a factor $y \in A^{+}$of length $k$ by $\bar{y}$. We say that a term obtained from $w_{x, j}(3)$ using these substitutions is irreducible when it is not possible to make more substitutions (that is, when there are no more occurrences of factors $y \in A^{+}$of length $k$ on the factors which resulted from substitutions on the words $w_{j}$ ). We choose an irreducible term and denote it by $w_{x, j}(4)$. This concludes the process of transformation of the word $w_{x, j}$.

We let $\widehat{w_{x, j}}=w_{x, j}(4)$. This concludes the process of transformation of the factors of $w_{x}$ in factorization (6.28). We then let

$$
\widehat{w_{x}}=\widehat{l_{x}} \widehat{w_{x, 0}} \widehat{c_{x, 1}} \cdots \widehat{c_{x, i_{x}}} \widehat{w_{x, i_{x}}} \widehat{r_{x}} .
$$

Definition 6.19 (labeling $\delta^{\prime}$ ) The labeling $\delta^{\prime}$ of $X$ by (rank $1 \kappa$-terms of) $\Omega_{A}^{\kappa} \mathbf{S}$ is defined by $\delta^{\prime}(x)=\widehat{w_{x}}$ for any $x \in X$.

That conditions (TT.1) to (TT.3) are verified also by non $\kappa$-variables is immediate. Therefore, to establish TT $\kappa$-reducibility of $\mathbf{L S l}$, it remains to show that $\delta^{\prime}$ is a $\kappa$ solution of $\mathcal{S}$ over $\mathbf{L S l}$ with respect to $(\gamma, \psi)$ and that condition (TT.5) is verified by all variables $x$ that are not $\kappa$-variables. This will be done in the next subsection.

\subsection{Proof of TT $\kappa$-reducibility}

As mentioned in Subsection 6.3, the labeling $\delta^{\prime}$ verifies $\psi \circ \delta^{\prime}=\gamma$ and, so, to prove that $\delta^{\prime}$ is a $\kappa$-solution of $\mathcal{S}$ over $\mathbf{L S l}$ with respect to $(\gamma, \psi)$, it suffices to show that pseudoidentity

$$
\delta^{\prime}\left(x_{i, 1}\right) \cdots \delta^{\prime}\left(x_{i, p_{i}}\right)=\delta^{\prime}\left(x_{i, p_{i}+1}\right) \cdots \delta^{\prime}\left(x_{i, q_{i}}\right)
$$

holds over LSl for every $i \in\{1, \ldots, h\}$. This is immediate if all variables $x_{i, j}$ are $\kappa$ variables. Indeed, in this case we have LSI $=\delta^{\prime}\left(x_{i, j}\right)=\delta\left(x_{i, j}\right)$, by condition (TT.4), which is valid as shown in the First Case. Therefore, since $\delta$ is a solution of $\mathcal{S}$ over LSl, so that $\mathbf{L S l}$ verifies $\delta\left(x_{i, 1}\right) \cdots \delta\left(x_{i, p_{i}}\right)=\delta\left(x_{i, p_{i}+1}\right) \cdots \delta\left(x_{i, q_{i}}\right)$, it is clear that (6.31) holds over LSI.

So, we assume that at least one variable $x_{i, j}$ is not a $\kappa$-variable (which means that at least two variables $x_{i, j_{1}}$ and $x_{i, j_{2}}$, with $j_{1} \leq p_{i}$ and $j_{2}>p_{i}$, are not $\kappa$-variables). Notice first that, by (6.10), (6.18) and (6.20) both sides of pseudoidentity (6.31) have the same essential prefix (i.e., $\widehat{l_{i, 1}}=\widehat{l_{i, p_{i}+1}}$ ) and the same essential suffix (i.e., $\widehat{r_{i, p_{i}}}=\widehat{r_{i, q_{i}}}$ ), which 
means that LI verifies (6.31). Therefore, by Proposition 4.8, it remains to prove that the $\kappa$-words

$$
z_{0}^{\prime}=\delta^{\prime}\left(x_{i, 1}\right) \cdots \delta^{\prime}\left(x_{i, p_{i}}\right)=\widehat{w_{i, 1}} \cdots \widehat{w_{i, p_{i}}}
$$

and

$$
z_{1}^{\prime}=\delta^{\prime}\left(x_{i, p_{i}+1}\right) \cdots \delta^{\prime}\left(x_{i, q_{i}}\right)=\widehat{w_{i, p_{i}+1}} \cdots \widehat{w_{i, q_{i}}}
$$

have the same non-periodic bi-infinite factors, for which it suffices to verify that they have the same essential factors, as already mentioned in Subsection 6.3.

We begin by observing that, since $\delta$ is a solution over LSI and $\equiv_{n}$ is a congruence on $\bar{\Omega}_{A} \mathbf{S}$, we deduce from Lemma 4.4 that $z_{0} \equiv_{n} z_{1}$, where $z_{0}=w_{i, 1} \cdots w_{i, p_{i}}$ and $z_{1}=$ $w_{i, p_{i}+1} \cdots w_{i, q_{i}}$. The observations in Lemma 6.20 below are simple consequences of this fact (see [15, Lemma 6.9] for a proof of a similar result). We say that an occurrence of a factor of length $3 k-1$ is relative-free in $z_{0}$ (resp. $z_{1}$ ) if it occurs in some $w_{i, j}$, with $1 \leq j \leq p_{i}$ (resp. $p_{i}+1 \leq j \leq q_{i}$ ), and it is free in $w_{i, j}$. Analogously, we say that a factor of $z_{0}$ (resp. $z_{1}$ ) is relative-marked if it is a marked factor in some $w_{i, j}$, with $1 \leq j \leq p_{i}$ (resp. $p_{i}+1 \leq j \leq q_{i}$ ).

Lemma 6.20 The following statements hold.

a) $z_{0}$ and $z_{1}$ have the same factors of length $3 k-1$ with relative-free occurrences.

b) $z_{0}$ and $z_{1}$ have the same relative-marked factors.

To prove that $z_{0}^{\prime}$ and $z_{1}^{\prime}$ have the same essential factors, we show that each essential factor $\bar{u} z \bar{v}$ of $z_{0}^{\prime}$ is also a factor of $z_{1}^{\prime}$ (the reverse follows by symmetry). The following cases may arise:

Case 1. $\bar{u} z \bar{v}$ overlaps two consecutive factors $\widehat{w_{i, j-1}}$ and $\widehat{w_{i, j}}$ of $z_{0}^{\prime}$, with $j \in\left\{2, \ldots, p_{i}\right\}$. Then $\bar{u} z \bar{v}=\widehat{c_{(i, j)}}$ for the center $c_{(i, j)}$ of the system. By (Sy.3) and (6.22), it is then clear that $\bar{u} z \bar{v}$ is also an essential factor of $z_{1}^{\prime}$.

Case 2. $\quad \bar{u} z \bar{v}$ occurs within some $\widehat{w_{i, \ell}}$ with $\ell \in\left\{1, \ldots, p_{i}\right\}$. We denote by $x$ the variable $x_{i, \ell}$ and notice that $\widehat{w_{x}}=\widehat{l_{x}} \widehat{w_{x, 0}} \widehat{c_{x, 1}} \cdots \widehat{c_{x, i_{x}}} \widehat{w_{x, i_{x}}} \widehat{r_{x}}$ was defined in $(6.30)$. The following subcases may arise:

Case 2.1. $\bar{u} z \bar{v}=\widehat{c_{x, j}}$ for some $j \in\left\{1, \ldots, i_{x}\right\}$. Since $c_{x, j}$ is a center of the system, the conclusion follows as in Case 1 .

Case 2.2. $\quad \bar{u} z \bar{v}$ overlaps $\widehat{l_{x}}$ and $\widehat{w_{x, 0}}$. Let $w_{x, 0}=w_{0} v_{1} w_{1} v_{2} \cdots v_{q} w_{q}$ be the marked factorization of $w_{x, 0}$.

If $w_{0}$ is the empty word, then $q \geq 1$ and it follows from Step 2.1 that $\bar{u} z \bar{v}=\widehat{l_{x}} \overline{v_{1,1}}$, where $v_{1,1}$ is the prefix of length $k$ of the marked factor $v_{1}$ of $w_{x, 0}$. By Lemma 5.6, $\left|v_{1}\right| \leq k^{\prime \prime}$. Now, notice that $l_{x} v_{1}$ is a prefix of $w_{x}$ of length at most $\left|l_{x}\right|+k^{\prime \prime}$, which is lower than $n$ by definition of $n$. By (Sy.3), there is some variable $y=x_{i, m}$, with $m \in\left\{p_{i}+1, \ldots, q_{i}\right\}$, such that $\mathbf{k}_{x}=\mathbf{k}_{y}$. Therefore, by definition of $w_{x}$ and $w_{y}, \delta(x), \delta(y), w_{x}$ and $w_{y}$ have all the same prefix of length $n$. Since $n$ is large enough, it follows that $v_{1}$ is not only a prefix of $w_{y, 0}$ but also a marked factor of $w_{y, 0}$. Moreover, by (6.18), $l_{x}=l_{y}$. Therefore $\bar{u} z \bar{v}=\widehat{l_{y}} \overline{v_{1,1}}$ also occurs in $z_{1}^{\prime}$.

If $w_{0}$ is not the empty word, then $\bar{u} z \bar{v}=\widehat{l_{x}} \overline{w_{0,1}}$ by Step 2.2, where $w_{0,1}$ is the prefix of length $k$ of $w_{0}$. This case can be treated as the above one. 
Case 2.3. $\quad \bar{u} z \bar{v}$ overlaps $\widehat{w_{x, i_{x}}}$ and $\widehat{r_{x}}$. This case is symmetric to Case 2.2.

Case 2.4. $\quad \bar{u} z \bar{v}$ overlaps $\widehat{w_{x, j-1}}$ and $\widehat{c_{x, j}}$, or it overlaps $\widehat{c_{x, j}}$ and $\widehat{w_{x, j}}$, for some $j \in$ $\left\{1, \ldots, i_{x}\right\}$. These cases are similar to Cases 2.2 and 2.3 , and they can be treated analogously using the fact that $\delta(x)$ and $w_{x}$ (resp. $z_{0}$ and $z_{1}$ ) have the same factors of length $n$.

Case 2.5. $\bar{u} z \bar{v}$ occurs within some $\widehat{w_{x, j}}$ with $j \in\left\{0, \ldots, i_{x}\right\}$. Consider the marked factorization $w_{x, j}=w_{0} v_{1} w_{1} v_{2} \cdots v_{q} w_{q}$. By definition of $\widehat{w_{x, j}}$ in Step 2, either $\bar{u} z \bar{v}=\widehat{v_{m}}$ and $u z v=v_{m}$ for some marked factor $v_{m}$ of $w_{x, j}$ (and $\bar{u} z \bar{v}$ was created in Step 2.1), or the corresponding occurrence of $u z v$ in $w_{x, j}$ is contained in a free occurrence (and $\bar{u} z \bar{v}$ was created in Step 2.3 or in Step 2.4). By Lemma 6.20, $u z v$ is a relative-marked factor of $z_{1}$ in the first case and is contained in a relative-free occurrence in $z_{1}$ in the second case. In both cases, it follows from Step 2 of the algorithm that $\bar{u} z \bar{v}$ is also an essential factor of $z_{1}$.

The above shows that $z_{0}^{\prime}$ and $z_{1}^{\prime}$ have the same essential factors, and concludes the proof that $\delta^{\prime}$ is a $\kappa$-solution of $\mathcal{S}$ over $\mathbf{L S l}$ with respect to $(\gamma, \psi)$.

To deduce the TT $\kappa$-reducibility of LSl, we finally show that condition (TT.5) is verified by each variable $x$ such that $x$ is not a $\kappa$-variable. The first part of this condition states that every prefix $u \in A^{*}$ of $\delta(x)$ with length at most $M$, is a prefix of $\delta^{\prime}(x)$ when $\delta^{\prime}(x)$ is regarded as a word over the alphabet $\bar{A}$. Or, this is a consequence of the fact that, by (6.30), $\delta^{\prime}(x)$ has $\widehat{l_{x}}$ as a prefix and, by (6.17), $\widehat{l_{x}}=p_{x} \overline{u_{\Theta}}$ with $p_{x} \in A^{+}$assumed (immediately after (6.15)) to be a word of length at least $M$.

To prove the second part of condition (TT.5), suppose that $\delta(x)=u \pi$ and $\delta(y)=v \rho$, where $y$ is another variable and $u, v \in A^{*}$ and $\pi, \rho \in \bar{\Omega}_{A} \mathbf{S}$ are such that $|u|,|v| \leq M$ and $\mathbf{L S I}=\pi=\rho$. We know from the first part that $\delta^{\prime}(x)=u \pi^{\prime}$ and $\delta^{\prime}(y)=v \rho^{\prime}$. We have to show that $\mathbf{L S l}=\pi^{\prime}=\rho^{\prime}$. By Lemma 4.4, since $\mathbf{L S l} \models \pi=\rho$, we may assume that $w_{x}=u w_{\pi}$ and $w_{y}=v w_{\rho}$ with $w_{\pi} \equiv_{n} w_{\rho}$. On the other hand, $\mathbf{K}=\pi=\rho$ and so, as $\mathbf{k}_{x}=u p_{\mathbf{K}}(\pi)$ and $\mathbf{k}_{y}=v p_{\mathbf{K}}(\rho)$, we deduce that $\mathbf{k}_{x}$ and $\mathbf{k}_{y}$ are confinal, i.e., that $\mathbf{k}_{x} \theta_{1} \mathbf{k}_{y}$. But, by (6.15), $\mathbf{k}_{x}=p_{x} p_{\Theta}$ and $\mathbf{k}_{y}=p_{y} p_{\Theta}$ where $\Theta$ is the $\theta_{1}$-class of $x$ and $y$. Hence, $p_{x}=u z$ and $p_{y}=v z$ for some word $z \in A^{*}$. By (6.17),$\widehat{l_{x}}=p_{x} \overline{u_{\Theta}}$ and $\widehat{l_{y}}=p_{y} \overline{u_{\Theta}}$, whence $z \overline{u_{\Theta}}$ is the essential prefix of both $\pi^{\prime}$ and $\rho^{\prime}$. That $\pi^{\prime}$ and $\rho^{\prime}$ also have the same essential suffix is a consequence of $(\underline{6.20})$ and of the fact that $\mathbf{D} \models \delta(x)=\delta(y)$. Finally, since $w_{\pi} \equiv_{n} w_{\rho}$, one can show that the words $w_{\pi}$ and $w_{\rho}$ have the same allowed centers, the same factors of length $3 k-1$ with relative-free occurrences and the same relative-marked factors. Whence, the proof that $\pi^{\prime}$ and $\rho^{\prime}$ have the same essential factors is analogous to the one above for $z_{0}^{\prime}$ and $z_{1}^{\prime}$. Therefore LSl $=\pi^{\prime}=\rho^{\prime}$, and condition (TT.5) is verified by $x$.

This establishes the TT $\kappa$-reducibility of LSl. The complete $\kappa$-reducibility of LSl, that is Theorem 6.1, is then obtained as a direct application of Proposition 6.13.

\section{Acknowledgements}

Both authors acknowledge partial support by the project PTDC/MAT/65481/2006. The work of the first author was supported, in part, by Fundação para a Ciência e a Tecnologia (FCT) through the Centro de Matemática da Universidade do Minho, which is partly funded by the European Community Fund FEDER. 


\section{References}

[1] D. Albert, R. Baldinger and J. Rhodes, The identity problem for finite semigroups (the undecidability of), J. Symbolic Logic 57 (1992) 179-192.

[2] J. Almeida, Finite semigroups and universal algebra, (World Scientific, Singapore, 1995). English translation.

[3] J. Almeida, Hyperdecidable pseudovarieties and the calculation of semidirect products, Int. J. Algebra and Computation 9 (1999) 241-261.

[4] J. Almeida, Dynamics of implicit operations and tameness of pseudovarieties of groups, Trans. Amer. Math. Soc. 354 (2002) 387-411.

[5] J. Almeida, Finite semigroups: An Introduction to a unified theory of pseudovarieties, in Semigroups, Algorithms, Automata and Languages, ed. G. Gomes (World Scientific, 2002), pp. 3-64.

[6] J. Almeida, J. C. Costa and M. Zeitoun, Tameness of pseudovariety joins involving R, Monatshefte für Mathematik 146 (2005), 89-111.

[7] J. Almeida, J. C. Costa and M. Zeitoun, Complete reducibility of pseudovarieties, in Proceedings of the International Conference Semigroups and Formal Languages, eds. J. André, M. Branco, V. Fernandes, J. Fountain, G. Gomes and J. Meakin (World Scientific, 2007), pp. 9-25.

[8] J. Almeida, J. C. Costa and M. Zeitoun, Complete reducibility of systems of equations with respect to R, Portugalice Mathematica 64 (2007), 445-508.

[9] J. Almeida and M. Delgado, Tameness of the pseudovariety of abelian groups, Int. J. Algebra and Computation 15 (2005), 327-338.

[10] J. Almeida and B. Steinberg, On the decidability of iterated semidirect products and applications to complexity, Proc. London Math. Soc. 80 (2000), 50-74.

[11] J. Almeida and B. Steinberg, Syntactic and global semigroup theory: a synthesis approach, Algorithmic problems in groups and semigroups (Lincoln, NE, 1998), Trends Math. (Birkhäuser Boston, Boston, MA, 2000), pp. 1-23.

[12] C. Ash, Inevitable graphs: a proof of the type II conjecture and some related decision procedures, Int. J. Algebra and Computation 1 (1991), 127-146.

[13] K. Auinger and B. Steinberg, On the extension problem for partial permutations, Proc. Amer. Math. Soc. 131 (2003), 2693-2703.

[14] J. C. Costa, Free profinite locally idempotent and locally commutative semigroups, Journal of Pure and Applied Algebra 163 (2001), 19-47.

[15] J. C. Costa and M. L. Teixeira, Tameness of the pseudovariety LSl, Int. J. Algebra and Computation 14 (2004), 627-654.

[16] T. Coulbois and A. Khélif, Equations in free groups are not finitely approximable, Proc. Amer. Math. Soc. 127 (1999), 963-965. 
[17] M. Lothaire, Algebraic combinatorics on words (Cambridge University Press, 2002).

[18] J. McCammond, Normal forms for free aperiodic semigroups, Int. J. Algebra and Computation 11 (2001), 581-625.

[19] J. Reiterman, The Birkhoff theorem for finite algebras, Algebra Universalis 14 (1982), 1-10.

[20] J. Rhodes, Undecidability, automata and pseudovarieties of semigroups, Int. J. Algebra and Computation 9 (1999), 455-473.

[21] J. Rhodes and B. Steinberg, The q-Theory of Finite Semigroups (Springer Monographs in Mathematics, Springer, 2008).

[22] B. Steinberg, On algorithmic problems for joins of pseudovarieties, Semigroup Forum 62 (2001), 1-40.

[23] B. Steinberg, A delay theorem for pointlikes, Semigroup Forum 63 (2001), 281-304. 